\title{
Fully Coupled Mean-Field Forward-Backward Stochastic Differential Equations and Stochastic Maximum Principle
}

\author{
Hui Min, ${ }^{1}$ Ying Peng, ${ }^{2}$ and Yongli Qin ${ }^{1,3}$ \\ ${ }^{1}$ School of Mathematics and Statistics, Shandong University, Weihai 264209, China \\ ${ }^{2}$ Department of Computer Science and Technology, Shandong University, Jinan 250101, China \\ ${ }^{3}$ BGP, CNPC, Zhuozhou 072750, China
}

Correspondence should be addressed to Yongli Qin; qinyongli8@gmail.com

Received 21 December 2013; Accepted 4 February 2014; Published 13 April 2014

Academic Editor: Michel Chipot

Copyright (C) 2014 Hui Min et al. This is an open access article distributed under the Creative Commons Attribution License, which permits unrestricted use, distribution, and reproduction in any medium, provided the original work is properly cited.

\begin{abstract}
We discuss a new type of fully coupled forward-backward stochastic differential equations (FBSDEs) whose coefficients depend on the states of the solution processes as well as their expected values, and we call them fully coupled mean-field forward-backward stochastic differential equations (mean-field FBSDEs). We first prove the existence and the uniqueness theorem of such meanfield FBSDEs under some certain monotonicity conditions and show the continuity property of the solutions with respect to the parameters. Then we discuss the stochastic optimal control problems of mean-field FBSDEs. The stochastic maximum principles are derived and the related mean-field linear quadratic optimal control problems are also discussed.
\end{abstract}

\section{Introduction}

Pardoux and Peng [1] in 1990 first introduced nonlinear classical backward stochastic differential equations (BSDEs). They proved the uniqueness and the existence of the solutions of nonlinear BSDEs under Lipschitz assumption. Since then the theory of BSDEs developed very fast and had found many applications, for example, in the stochastic control and partial differential equations. On the other hand, those stochastic Hamilton systems, derived from the stochastic maximum principle of stochastic optimal control problems, are forwardbackward stochastic differential equations (FBSDEs).

The theory of fully coupled FBSDEs develops also very dynamically. There are many works on the existence and the uniqueness of solutions of fully coupled FBSDEs. Antonelli [2] first proved the existence and the uniqueness of solutions of fully coupled FBSDEs driven by Brownian motion on a small time interval with the fixed point theorem. There are also many other methods to study fully coupled FBSDEs on an arbitrarily given time interval, mainly three methods. One is "four-step scheme" approach (see Ma et al. [3]) which combines PDE methods and probability methods. The authors proved the existence and the uniqueness for fully coupled FBSDEs on an arbitrarily given time interval, but they required the diffusion coefficients to be nondegenerate and deterministic. Another one is purely probabilistic continuation method; refer to Hu and Peng [4], Pardoux and Tang [5], Peng and $\mathrm{Wu}$ [6], Yong [7], and so on. Another method is inspired by the numerical approaches for some linear FBSDEs (see Delarue and Menozzi [8] and Zhang [9]). There are also other methods; see Ma et al. [10]. For more details about fully coupled FBSDEs, the readers also refer to Ma and Yong [11] or Yong [7] and the references therein.

On the other hand, the theory of the modern optimal control has been developed widely since Pontryagin et al's work [12] about the maximum principle and Bellman's work [13] on the dynamic programming approach. Later there have been a lot of works on the stochastic maximum principle; see, for example, Kushner [14, 15], Bensoussan [16], Haussmann [17], Peng [18], Wu [19], and so on. Wu [19] discussed the stochastic maximum principle for the fully coupled FBSDEs. Recently the methods of mean-field are used in various fields, such as in Finance, Chemistry, and Game Theory. The meanfield backward stochastic differential equations (mean-field BSDEs) were introduced by [20]; for more properties about 
mean-field BSDEs we refer to [21]. There are also many works on stochastic maximum principle for SDEs of mean-field type; see Andersson and Djehiche [22], Buckdahn et al. [23], Li [24], Bensoussan et al. [25], and so on. For more details we may refer to Yong [7].

In this paper, we consider the following fully coupled mean-field forward-backward stochastic differential equations (mean-field FBSDEs in short):

$$
\begin{gathered}
d x(t)=E^{\prime}[f(t, \chi(t))] d t+E^{\prime}[\sigma(t, \chi(t))] d B_{t}, \\
-d y(t)=E^{\prime}[g(t, \chi(t))] d t-z(t) d B_{t}, \\
x(0)=a, y(T)=E^{\prime}\left[\Phi\left(x(T),(x(T))^{\prime}\right)\right],
\end{gathered}
$$

where $\chi(t):=\left(x(t), y(t), z(t),(x(t))^{\prime},(y(t))^{\prime},(z(t))^{\prime}\right),(x(\cdot)$, $y(\cdot), z(\cdot))$ take values in $\mathbf{R}^{n} \times \mathbf{R}^{m} \times \mathbf{R}^{m \times d} ; f, \sigma, g, \Phi$ are mappings with appropriate dimensions which are $\overline{\mathbb{F}}$-progressively measurable. The time duration $T \geq 0$ is an arbitrarily fixed number. Our aim is first to find a triplet $\mathbb{F}$-adapted processes $(x(\cdot), y(\cdot), z(\cdot))$ satisfying (1) and then study the stochastic maximum principle of mean-field FBSDEs with controls. For more works we refer to Qin [26].

In Section 2, we introduce the mean-field BSDEs. In Section 3, we prove the existence and the uniqueness of solution of mean-field FBSDE by the continuation method. In Section 4, we give the continuity of solutions of meanfield FBSDE with respect to the parameters and also give an example to show that our monotonicity conditions are necessary. In Section 5 we study the stochastic maximum principle for mean-field FBSDEs with controls and obtain the necessary condition of the stochastic maximum principle. In Section 6 we discuss mean-field backward stochastic linear quadratic optimal control problem as an example.

\section{Preliminaries}

Let $(\Omega, \mathscr{F}, P)$ be a complete probability space with a standard $d$-dimensional Brownian motion $B=\left(B_{t}\right)_{t \geq 0}$, and let $\mathscr{F}_{t}$ be the natural filtration generated by $B$ and augmented by all Pnull sets (i.e., $\mathscr{F}_{t}=\sigma\left\{B_{s}: 0 \leq s \leq t\right\} \bigvee \mathcal{N}_{P}, t \geq 0$, where $\mathcal{N}_{P}$ is the set of all P-null subsets). $T>0$ is the fixed time horizon. $\mathbb{F}=\left\{\mathscr{F}_{t}, t \geq 0\right\}$.

Let $(\bar{\Omega}, \bar{F}, \bar{P})=(\Omega \times \Omega, \mathscr{F} \otimes \mathscr{F}, P \otimes P)$ be the (noncompleted) product of $(\Omega, \mathscr{F}, P)$ with itself. This product space is endowed with the filtration $\overline{\mathbb{F}}=\left\{\overline{\mathscr{F}}_{t}=\mathscr{F} \otimes \mathscr{F}_{t}, 0 \leq\right.$ $t \leq T\}$. A random variable $\xi \in L^{0}\left(\Omega, \mathscr{F}, P ; \mathbb{R}^{n}\right)$ originally defined on $\Omega$ is extended canonically to $\bar{\Omega}: \xi^{\prime}\left(\omega^{\prime}, \omega\right)=$ $\xi\left(\omega^{\prime}\right),\left(\omega^{\prime}, \omega\right) \in \bar{\Omega}=\Omega \times \Omega$. For any $\theta \in L^{1}(\bar{\Omega}, \overline{\mathscr{F}}, \bar{P})$ the variable $\theta(\cdot, \omega): \Omega \rightarrow \mathbb{R}$ is in $L^{1}(\Omega, \mathscr{F}, P), P(d \omega)$-a.s., and its expectation is denoted by

$$
E^{\prime}[\theta(\cdot, \omega)]=\int_{\Omega} \theta\left(\omega^{\prime}, \omega\right) P\left(d \omega^{\prime}\right) .
$$

We notice that $E^{\prime}[\theta]=E^{\prime}[\theta(\cdot, \omega)] \in L^{1}(\Omega, \mathscr{F}, P)$ and

$$
\bar{E}[\theta]\left(=\int_{\bar{\Omega}} \theta d \bar{P}=\int_{\Omega} E^{\prime}[\theta(\cdot, \omega)] P(d \omega)\right)=E\left[E^{\prime}[\theta]\right] .
$$

The generator of our mean-field BSDE is a mapping:

$$
\begin{aligned}
f=f\left(t, \omega, \omega^{\prime}, y, z, \tilde{y}, \tilde{z}\right): & {[0, T] \times \bar{\Omega} \times \mathbb{R}^{m} } \\
& \times \mathbb{R}^{m \times d} \times \mathbb{R}^{m} \\
& \times \mathbb{R}^{m \times d} \longrightarrow \mathbb{R}^{m},
\end{aligned}
$$

which is $\overline{\mathbb{F}}$-progressively measurable, for all $(y, z, \tilde{y}, \tilde{z})$, and satisfies the following assumptions.

We assume the following.

(i) $f(t, y, z, \tilde{y}, \widetilde{z})$ is uniformly Lipschitz with respect to $y, z, \tilde{y}, \widetilde{z}$

(ii) $\quad f(\cdot, 0,0,0,0) \in M_{\overrightarrow{\mathbb{F}}}^{2}\left(0, T ; \mathbb{R}^{m}\right)$; that is, $f(\cdot, 0,0,0,0)$ is $\mathbb{R}^{m}$-valued $\overline{\mathbb{F}}$-progressively measurable and $\bar{E}\left[\int_{0}^{T}|f(t, 0,0,0,0)|^{2} d t\right]<+\infty$.

Lemma 1. Let (H2.1) hold, for any random variable $\xi \in$ $L^{2}\left(\Omega, \mathscr{F}_{T}, P ; \mathbb{R}^{m}\right)$; the mean-field BSDE

$$
\begin{aligned}
Y_{t}= & \xi+\int_{t}^{T} E^{\prime}\left[f\left(s, Y_{s}, Z_{s},\left(Y_{s}\right)^{\prime},\left(Z_{s}\right)^{\prime}\right)\right] d s \\
& -\int_{t}^{T} Z_{s} d B_{s}, \quad 0 \leq t \leq T
\end{aligned}
$$

has a unique solution $(Y, Z) \in S_{\mathbb{F}}^{2}\left(0, T ; \mathbb{R}^{m}\right) \times M_{\mathbb{F}}^{2}\left(0, T ; \mathbb{R}^{m \times d}\right)$; that is, $Y$ is $\mathbb{R}^{m}$-valued $\mathbb{F}$-adapted continuous process and $E\left[\sup _{0 \leq t \leq T}\left|Y_{t}\right|^{2}\right]<\infty ; Z$ is $\mathbb{R}^{m \times d}$-valued $\mathbb{F}$-progressively measurable process and $E\left[\int_{0}^{T}\left|Z_{t}\right|^{2} d t\right]<\infty$.

For the proof, the readers may refer to [20].

Remark 2. From the above notions, the generator of the above mean-feld BSDE has to be understood as follows:

$$
\begin{aligned}
E^{\prime} & {\left[f\left(s, Y_{s}, Z_{s},\left(Y_{s}\right)^{\prime},\left(Z_{s}\right)^{\prime}\right)\right](\omega) } \\
& =E^{\prime}\left[f\left(s, Y_{s}(\omega), Z_{s}(\omega),\left(Y_{s}\right)^{\prime},\left(Z_{s}\right)^{\prime}\right)\right] \\
& =\int_{\Omega} f\left(\omega^{\prime}, \omega, s, Y_{s}(\omega), Z_{s}(\omega), Y_{s}\left(\omega^{\prime}\right), Z_{s}\left(\omega^{\prime}\right)\right) P\left(d \omega^{\prime}\right),
\end{aligned}
$$

Remark 3. If we assume that

(i) $b(\cdot, x, \tilde{x})$ and $\sigma(\cdot, x, \tilde{x})$ are $\overline{\mathbb{F}}$-progressively measurable continuous processes, for all $x, \tilde{x} \in \mathbb{R}^{n}$ and there exists some constant $C>0$ such that $|b(t, x, \tilde{x})|+$ $|\sigma(t, x, \tilde{x})| \leq C(1+|x|+|\tilde{x}|)$, a.s. , $0 \leq t \leq T, x, \tilde{x} \in \mathbb{R}^{n}$,

(ii) $b$ and $\sigma$ are Lipschitz in $x, \tilde{x}$, 
then, for any random variable $\zeta \in L^{2}\left(\Omega, \mathscr{F}_{T}, P ; \mathbb{R}^{n}\right)$, the following mean-field SDE which is also the Mckean-Vlasov SDE has a unique adapted solution $X \in S_{\mathbb{F}}^{2}\left(0, T ; \mathbb{R}^{n}\right)$ :

$$
\begin{array}{r}
X_{t}=\zeta+\int_{0}^{t} E^{\prime}\left[b\left(s, X_{s},\left(X_{s}\right)^{\prime}\right)\right] d s \\
+\int_{0}^{t} E^{\prime}\left[\sigma\left(s, X_{s},\left(X_{s}\right)^{\prime}\right)\right] d B_{s} \\
0 \leq t \leq T .
\end{array}
$$

For more details, the reader may refer to, for example, [20] or [24].

\section{Mean-Field FBSDE: Existence and Uniqueness}

We consider the following fully coupled mean-field forwardbackward stochastic differential equations:

$$
\begin{gathered}
d x(t)=E^{\prime}[f(t, \chi(t))] d t+E^{\prime}[\sigma(t, \chi(t))] d B_{t}, \\
-d y(t)=E^{\prime}[g(t, \chi(t))] d t-z(t) d B_{t}, \\
x(0)=a, \quad y(T)=E^{\prime}\left[\Phi\left(x(T),(x(T))^{\prime}\right)\right],
\end{gathered}
$$

where

$$
\begin{aligned}
\chi(t)=\left(x(t), y(t), z(t),(x(t))^{\prime},(y(t))^{\prime},(z(t))^{\prime}\right), \\
f:[0, T] \times \bar{\Omega} \times \mathbb{R}^{n} \times \mathbb{R}^{m} \times \mathbb{R}^{m \times d} \\
\times \mathbb{R}^{n} \times \mathbb{R}^{m} \times \mathbb{R}^{m \times d} \longrightarrow \mathbb{R}^{n}, \\
\sigma:[0, T] \times \bar{\Omega} \times \mathbb{R}^{n} \times \mathbb{R}^{m} \times \mathbb{R}^{m \times d} \\
\times \mathbb{R}^{n} \times \mathbb{R}^{m} \times \mathbb{R}^{m \times d} \longrightarrow \mathbb{R}^{n \times d}, \\
g:[0, T] \times \bar{\Omega} \times \mathbb{R}^{n} \times \mathbb{R}^{m} \times \mathbb{R}^{m \times d} \\
\times \mathbb{R}^{n} \times \mathbb{R}^{m} \times \mathbb{R}^{m \times d} \longrightarrow \mathbb{R}^{m}, \\
\Phi: \bar{\Omega} \times \mathbb{R}^{n} \times \mathbb{R}^{n} \longrightarrow \mathbb{R}^{m} .
\end{aligned}
$$

Remark 4. In $\mathrm{Li}$ [24], the author studied the stochastic maximum principle in mean-field controls; the related feedback control system takes a special case of the mean-field FBSDE (8).

Given an $m \times n$ full-rank matrix $G$. We use the following notations:

$$
\begin{aligned}
& \lambda=\left(\begin{array}{c}
x \\
y \\
z
\end{array}\right), \quad \tilde{\lambda}=\left(\begin{array}{c}
\tilde{x} \\
\tilde{y} \\
\tilde{z}
\end{array}\right), \\
& \mathbf{A}(\mathbf{t}, \lambda, \tilde{\lambda})=\left(\begin{array}{c}
-G^{T} g \\
G f \\
G \sigma
\end{array}\right)(t, \lambda, \tilde{\lambda}),
\end{aligned}
$$

where $G \sigma=\left(G \sigma_{1}, \ldots, G \sigma_{d}\right)$. We use the standard inner product and Euclidean norm in $\mathbb{R}^{m \times d}$.
Definition 5. A triple of processes $(X, Y, Z)$ is called an adapted solution of mean-field $\operatorname{FBSDE}(8)$, if $(X, Y, Z) \in$ $M_{\mathbb{F}}^{2}\left(0, T ; \mathbb{R}^{n} \times \mathbb{R}^{m} \times \mathbb{R}^{m \times d}\right)$, and satisfies mean-field FBSDE (8).

We assume the following.

(i) $A(t, \lambda, \widetilde{\lambda})$ is uniformly Lipschitz with respect to $\lambda, \widetilde{\lambda}$;

(ii) for each $\lambda, \tilde{\lambda}, A(\cdot, \lambda, \tilde{\lambda})$ is in $M_{\overline{\mathbb{F}}}^{2}(0, T)$;

(iii) $\Phi(x, \tilde{x})$ is uniformly Lipchitz with respect to $x, \tilde{x} \in$ $\mathbb{R}^{n}$

(iv) for each $x, \tilde{x}, \Phi(x, \tilde{x})$ is in $L^{2}\left(\bar{\Omega}, \overline{\mathscr{F}}_{T}, \bar{P}\right)$;

(v) the coefficients $(f, \sigma, g)$ are uniformly Lipschitz to $(x, y, z, \tilde{x}, \tilde{y}, \widetilde{z})$.

We also need the following monotonicity assumptions.

(i) $\langle A(t, \lambda, \tilde{\lambda})-A(t, \bar{\lambda}, \tilde{\lambda}), \lambda-\bar{\lambda}\rangle \leq-\beta_{1}|\widehat{x}|^{2}-\beta_{2}\left(|\widehat{y}|^{2}+|\widehat{z}|^{2}\right)$;

(ii) $\langle\Phi(x, \tilde{x})-\Phi(\bar{x}, \tilde{x}), G(x-\bar{x})\rangle \geq \mu_{1}|\widehat{x}|^{2}$;

$\forall \lambda=(x, y, z), \quad \tilde{\lambda}=(\tilde{x}, \tilde{y}, \tilde{z}), \quad \bar{\lambda}=(\bar{x}, \bar{y}, \bar{z})$,

$\widehat{l}=l-\bar{l}$, where $l=x, y, z, \tilde{x}, \tilde{y}, \tilde{z}$, respectively.

$\beta_{1}, \beta_{2}$, and $\mu_{1}$ are given nonnegative constants with $\beta_{1}-L_{A} \geq$ $0, \beta_{2}-L_{A} \geq 0$ (the equalities cannot be established at the same time), and $\mu_{1}-L_{\Phi} \lambda_{1}>0 ; L_{A}, L_{\Phi}$ are the Lipschitz constants of $A, \Phi$ with respect to $\tilde{\lambda}, \tilde{x}$, respectively; and $\lambda_{1}$ satisfies $|G \widehat{l}(T)| \leq \lambda_{1}|\widehat{l}(T)|$.

Or we need the following.

(i) $\langle A(t, \lambda, \tilde{\lambda})-A(t, \bar{\lambda}, \tilde{\lambda}), \lambda-\bar{\lambda}\rangle \geq \beta_{1}|\widehat{x}|^{2}+\beta_{2}\left(|\widehat{y}|^{2}+|\widehat{z}|^{2}\right)$;

(ii) $\langle\Phi(x, \tilde{x})-\Phi(\bar{x}, \tilde{x}), G(x-\bar{x})\rangle \leq-\mu_{1}|\widehat{x}|^{2}$;

$\forall \lambda=(x, y, z), \quad \tilde{\lambda}=(\tilde{x}, \tilde{y}, \tilde{z}), \quad \bar{\lambda}=(\bar{x}, \bar{y}, \bar{z})$,

$\widehat{l}=l-\bar{l}$, where $l=x, y, z, \tilde{x}, \tilde{y}, \tilde{z}$, respectively.

$\beta_{1}, \beta_{2}$, and $\mu_{1}$ are given nonnegative constants with $\beta_{1}-L_{A} \geq$ $0, \beta_{2}-L_{A} \geq 0$ (the equalities cannot be established at the same time), and $\mu_{1}-L_{\Phi} \lambda_{1}>0$.

Then we have the following two main results in this section.

Theorem 6. One assumes that (H3.1) and (H3.2) hold; then mean-field FBSDE (8) has a unique adapted solution $(X, Y, Z)$.

Remark 7. Similarly, if (H3.1) and (H3.3) hold, then meanfield FBSDE (8) has a unique adapted solution $(X, Y, Z)$.

Proof. We first prove the uniqueness. Let $\lambda(t)=(x(t), y(t)$, $z(t))$ and $\bar{\lambda}(t)=(\bar{x}(t), \bar{y}(t), \bar{z}(t))$ be two solutions of (8). We 
set $\widehat{l}=l-\bar{l}$, where $l=x, y, z, \tilde{x}, \tilde{y}, \widetilde{z}$, respectively. Applying Itô's formula to $\langle G \widehat{x}(s), \widehat{y}(s)\rangle$, we get

$$
\begin{aligned}
E\left\langle\left( E^{\prime}\left[\Phi\left(x(T),(x(T))^{\prime}\right)\right]\right.\right. \\
\left.\left.-E^{\prime}\left[\Phi\left(\bar{x}(T),(\bar{x}(T))^{\prime}\right)\right]\right), G(x(T)-\bar{x}(T))\right\rangle \\
=E \int_{0}^{T}\left\langle\left( E^{\prime}\left[A\left(t, \lambda(t),(\lambda(t))^{\prime}\right)\right]\right.\right. \\
\left.\left.\quad-E^{\prime}\left[A\left(t, \bar{\lambda}(t),(\bar{\lambda}(t))^{\prime}\right)\right]\right), \lambda(t)-\bar{\lambda}(t)\right\rangle d t .
\end{aligned}
$$

From (H3.2) the monotonicity assumptions of $\Phi$ and $A$, we get

$$
\begin{aligned}
\left(\mu_{1}\right. & \left.-L_{\Phi} \lambda_{1}\right) E\left[|\widehat{x}(T)|^{2}\right] \\
\leq & -E \int_{0}^{T}\left[\beta_{1}|\widehat{x}(t)|^{2}+\beta_{2}\left(|\widehat{y}(t)|^{2}+|\widehat{z}(t)|^{2}\right)\right] d t \\
& +L_{A} E \int_{0}^{T}\left[|\widehat{x}(t)|^{2}+|\widehat{y}(t)|^{2}+|\widehat{z}(t)|^{2}\right] d t .
\end{aligned}
$$

When $\beta_{1}-L_{A}>0, \beta_{2}-L_{A} \geq 0, \mu_{1}-L_{\Phi} \lambda_{1}>0,|\widehat{x}(t)|^{2}=$ $0, d s d P$-a.e. In this case we have $\widehat{x}(t)=0, P$-a.s., for all $t \in[0, T]$. Thus, $\Phi\left(x(T),(x(T))^{\prime}\right)=\Phi\left(\bar{x}(T),(\bar{x}(T))^{\prime}\right), \bar{P}$-a.s. Therefore, from Lemma 1 it follows that $y(t)=\bar{y}(t), P$-a.s. and $z(t)=\bar{z}(t), P$-a.s.a.e. When $\beta_{1}-L_{A}=0, \beta_{2}-L_{A}>$ $0, \mu_{1}-L_{\Phi} \lambda_{1}>0$, thus $y(t)=\bar{y}(t), z(t)=\bar{z}(t), x(T)=\bar{x}(T)$, $P$-a.s.a.e. From the uniqueness of solutions of McKean-Vlasov equations (refer to [20] or Remark 2), we get $x(t)=\bar{x}(t), P$ a.s., for all $t \in[0, T]$.

For the existence, we need to combine the above techniques and an a priori estimate to construct a contraction mapping. For this we first prove the following lemma.

For $\beta_{1}-L_{A} \geq 0, \beta_{2}-L_{A} \geq 0, \mu_{1}-L_{\Phi} \lambda_{1}>0$ (the equalities cannot be established at the same time). We consider the following mean-field FBSDEs parameterized by $\alpha \in[0,1]$ :

$$
\begin{gathered}
d x^{\alpha}(t) \\
=\left[\alpha E^{\prime}\left[f\left(t, \chi^{\alpha}(t)\right)\right]+E^{\prime}[\phi(t)]\right] d t \\
+\left[\alpha E^{\prime}\left[\sigma\left(t, \chi^{\alpha}(t)\right)\right]+E^{\prime}[\psi(t)]\right] d B_{t}, \\
-d y^{\alpha}(t) \\
=\left[(1-\alpha) \beta_{1} G x^{\alpha}(t)+\alpha E^{\prime}\left[g\left(t, \chi^{\alpha}(t)\right)\right]+E^{\prime}[\gamma(t)]\right] d t \\
-z^{\alpha}(t) d B_{t}, \quad \\
x^{\alpha}(0)=a, \\
y^{\alpha}(T)=\alpha E^{\prime}\left[\Phi\left(x^{\alpha}(T),\left(x^{\alpha}(T)\right)^{\prime}\right)\right] \\
+(1-\alpha) G x^{\alpha}(T)+\xi,
\end{gathered}
$$

where $\chi^{\alpha}(t)=\left(x^{\alpha}(t), y^{\alpha}(t), z^{\alpha}(t),\left(x^{\alpha}(t)\right)^{\prime},\left(y^{\alpha}(t)\right)^{\prime},\left(z^{\alpha}(t)\right)^{\prime}\right)$ and $\phi, \psi$, and $\gamma$ are given processes in $M_{\overline{\mathbb{F}}}^{2}(0, T)$ with values in $\mathbb{R}^{n}, \mathbb{R}^{n \times d}$, and $\mathbb{R}^{m}$, respectively. $\xi \in L^{2}\left(\Omega, \mathscr{F}_{T}, P\right)$. Obviously, when $\alpha=1$, the existence of (15) implies that of (8). From the existence and the uniqueness of Mckean-Vlasov equation and mean-field BSDE, (15) has a unique solution when $\alpha=0$. The following lemma is needed.

Lemma 8. One assumes that (H3.1) and (H3.2) hold. If for an $\alpha_{0} \in[0,1)$ there exists a solution $\left(x^{\alpha_{0}}, y^{\alpha_{0}}, z^{\alpha_{0}}\right)$ of $(15)$, then there exists a positive constant $\delta_{0}$ such that for each $\delta \in$ $\left[0, \delta_{0}\right]$ there exists a solution $\left(x^{\alpha_{0}+\delta}, y^{\alpha_{0}+\delta}, z^{\alpha_{0}+\delta}\right)$ of mean-field FBSDE (15) for $\alpha=\alpha_{0}+\delta$.

Proof. Since for every $\phi \in M_{\mathbb{\mathbb { F }}}^{2}\left(0, T ; \mathbb{R}^{n}\right), \gamma \in M_{\mathbb{\mathbb { F }}}^{2}(0, T$; $\left.\mathbb{R}^{m}\right), \psi \in M_{\overline{\mathbb{F}}}^{2}\left(0, T, \mathbb{R}^{n \times d}\right), \alpha_{0} \in[0,1)$, there exists a (unique) solution of (15); for each $x(T) \in L^{2}\left(\Omega, \mathscr{F}_{T}, P\right)$ and a triple $(\lambda(t))_{0 \leq t \leq T}=(x(t), y(t), z(t))_{0 \leq t \leq T} \in M_{\mathbb{F}}^{2}\left(0, T ; \mathbb{R}^{n+m+m \times d}\right)$ there exists a unique triple

$$
\begin{aligned}
(\Lambda(t))_{0 \leq t \leq T} & =(X(t), Y(t), Z(t))_{0 \leq t \leq T} \\
& \in M_{\mathbb{F}}^{2}\left(0, T ; \mathbb{R}^{n+m+m \times d}\right)
\end{aligned}
$$

satisfying the following mean-field FBSDEs:

$$
\begin{aligned}
d X(t)= & {\left[\alpha_{0} E^{\prime}\left[f\left(t, \Lambda(t),(\Lambda(t))^{\prime}\right)\right]\right.} \\
& \left.+\delta E^{\prime}\left[f\left(t, \lambda(t),(\lambda(t))^{\prime}\right)\right]+E^{\prime}[\phi(t)]\right] d t \\
+ & {\left[\alpha_{0} E^{\prime}\left[\sigma\left(t, \Lambda(t),(\Lambda(t))^{\prime}\right)\right]\right.} \\
& \left.+\delta E^{\prime}\left[\sigma\left(t, \lambda(t),(\lambda(t))^{\prime}\right)\right]+E^{\prime}[\psi(t)]\right] d B_{t}, \\
-d Y(t) \quad & {\left[\left(1-\alpha_{0}\right) \beta_{1} G X(t)+\alpha_{0} E^{\prime}\left[g\left(t, \Lambda(t),(\Lambda(t))^{\prime}\right)\right]\right.} \\
+ & \delta\left(-\beta_{1} G x(t)+E^{\prime}\left[g\left(t, \lambda(t),(\lambda(t))^{\prime}\right)\right]\right) \\
+ & \left.E^{\prime}[\gamma(t)]\right] d t \\
- & Z(t) d B_{t}, \\
& \quad X(0)=a, \\
Y(T) & \quad \\
= & \alpha_{0} E^{\prime}\left[\Phi\left(X(T),(X(T))^{\prime}\right)\right]+\left(1-\alpha_{0}\right) G X(T) \\
+ & \delta\left(E^{\prime}\left[\Phi\left(x(T),(x(T))^{\prime}\right)\right]-G x(T)\right)+\xi .
\end{aligned}
$$

We want to prove that if $\delta$ is small enough, the mapping defined by $I_{\alpha_{0}+\delta}(\lambda \times x(T))=\Lambda \times X(T): M_{\mathbb{F}}^{2}\left(0, T ; \mathbb{R}^{n+m+m \times d}\right) \times$ $L^{2}\left(\Omega, \mathscr{F}_{T}, \mathbf{P}\right) \mapsto M_{\mathbb{F}}^{2}\left(0, T ; \mathbb{R}^{n+m+m \times d}\right) \times L^{2}\left(\Omega, \mathscr{F}_{T}, \mathbf{P}\right)$ is a contraction. Let $\bar{\lambda}=(\bar{x}, \bar{y}, \bar{z}) \in M_{\mathbb{F}}^{2}\left(0, T ; \mathbb{R}^{n+m+m \times d}\right)$ and $\bar{\Lambda} \times \bar{X}(T)=I_{\alpha_{0}+\delta}(\bar{\lambda} \times \bar{x}(T))$. We define $\hat{\lambda}=(\widehat{x}, \hat{y}, \widehat{z})=(x-$ $\bar{x}, y-\bar{y}, z-\bar{z}), \quad \hat{\lambda}^{\prime}=\left(\hat{x}^{\prime}, \hat{y}^{\prime}\right)=\left(x^{\prime}-\bar{x}^{\prime}, y^{\prime}-\bar{y}^{\prime}, z^{\prime}-\bar{z}^{\prime}\right), \hat{\Lambda}=$ $(\widehat{X}, \widehat{Y}, \widehat{Z})=(X-\bar{X}, Y-\bar{Y}, Z-\bar{Z}), \quad \widehat{\Lambda}^{\prime}=\left(\widehat{X}^{\prime}, \widehat{Y}^{\prime}, \widehat{Z}^{\prime}\right)=$ $\left(X^{\prime}-\bar{X}^{\prime}, Y^{\prime}-\bar{Y}^{\prime}, Z^{\prime}-\bar{Z}^{\prime}\right)$. 
Applying Itô’s formula to $\langle G \widehat{X}(t), \widehat{Y}(t)\rangle$, it yields

$$
\begin{gathered}
E\left\langle\alpha_{0} E^{\prime}\left[\Phi\left(X(T),(X(T))^{\prime}\right)-\Phi\left(\bar{X}(T),(\bar{X}(T))^{\prime}\right)\right]\right. \\
+\left(1-\alpha_{0}\right) G \widehat{X}(T) \\
+\delta\left(E^{\prime}\left[\Phi\left(x(T),(x(T))^{\prime}\right)-\Phi\left(\bar{x}(T),(\bar{x}(T))^{\prime}\right)\right]\right. \\
-G \widehat{x}(T)), G \widehat{X}(T)\rangle \\
=E \int_{0}^{T}\left\{\left\langle\alpha _ { 0 } E ^ { \prime } \left[A\left(t, \Lambda(t),(\Lambda(t))^{\prime}\right)\right.\right.\right. \\
\left.\left.-A\left(t, \bar{\Lambda}(t),(\bar{\Lambda}(t))^{\prime}\right)\right], \widehat{\Lambda}(t)\right\rangle \\
-\left(1-\alpha_{0}\right) \beta_{1}\langle G \widehat{X}(t), G \widehat{X}(t)\rangle \\
+\delta E^{\prime}\left[\beta_{1}\langle G \widehat{X}(t), G \widehat{x}(t)\rangle+\langle G \widehat{X}(t), \widehat{g}(t)\rangle\right. \\
+\left\langle G^{T} \widehat{Y}(t), \widehat{f}(t)\right\rangle \\
\left.\left.+\left\langle G^{T} \widehat{Z}(t), \widehat{\sigma}(t)\right\rangle\right]\right\} d t,
\end{gathered}
$$

where $\widehat{f}(t)=f\left(t, \lambda(t),(\lambda(t))^{\prime}\right)-f\left(t, \bar{\lambda}(t),(\bar{\lambda}(t))^{\prime}\right), \widehat{g}(t)=-g$ $\left(t, \lambda(t),(\lambda(t))^{\prime}\right)+g\left(t, \bar{\lambda}(t),(\bar{\lambda}(t))^{\prime}\right), \widehat{\sigma}(t)=\sigma\left(t, \lambda(t),(\lambda(t))^{\prime}\right)-$ $\sigma\left(t, \bar{\lambda}(t),(\bar{\lambda}(t))^{\prime}\right)$.

From (H3.1) and (H3.2), we know that if $\beta_{1}-L_{A}=0, \mu_{1}-$ $L_{\Phi} \lambda_{1}>0$, then $\beta_{2}-L_{A}>0$. Then, we have

$$
\begin{aligned}
E\left[\int _ { 0 } ^ { T } \left(|\widehat{Y}(t)|^{2}\right.\right. & \left.\left.+|\widehat{Z}(t)|^{2}\right) d t\right] \\
\leq \delta C_{2} E\left\{\int_{0}^{T}|\widehat{\Lambda}(t)|^{2} d t+|\widehat{X}(T)|^{2}\right. & \\
& \left.+\int_{0}^{T}|\widehat{\lambda}(t)|^{2} d t+|\widehat{x}(T)|^{2}\right\} .
\end{aligned}
$$

On the other hand, from standard technique to the forward equation for $\widehat{X}(t)=X(t)-\bar{X}(t)$, we get

$$
\begin{aligned}
\sup _{0 \leq t \leq T} E & {\left[|\widehat{X}(t)|^{2}\right] } \\
\leq & \delta C_{2} E\left[\int_{0}^{T}|\widehat{\lambda}(t)|^{2} d t\right] \\
& +C_{2} E\left[\int_{0}^{T}\left[|\widehat{Y}(t)|^{2}+|\widehat{Z}(t)|^{2}\right] d t\right] .
\end{aligned}
$$

From the above two estimates, we have

$$
\begin{aligned}
& E\left\{\int_{0}^{T}|\widehat{\Lambda}(t)|^{2} d t+|\widehat{X}(T)|^{2}\right\} \\
& \leq \bar{C} \delta E\left\{\int_{0}^{T}|\widehat{\lambda}(t)| d t+|\widehat{x}(T)|^{2}\right\} .
\end{aligned}
$$

Here the constant $\bar{C}$ depends on the Lipschitz constants, $\lambda_{1}, \beta_{1}, \beta_{2}$, and $T$.

If $\beta_{1}-L_{A}>0, \mu_{1}-L_{\Phi} \lambda_{1}>0$, then $\beta_{2}-L_{A} \geq 0$. Then, we have

$$
\begin{aligned}
E\left[|\widehat{X}(T)|^{2}\right] & +E\left[\int_{0}^{T}|\widehat{X}(t)|^{2} d t\right] \\
\leq \delta C_{1} E\left\{\int_{0}^{T}|\widehat{\Lambda}(t)|^{2} d t\right. & \\
& \left.+\int_{0}^{T}|\widehat{\lambda}(t)|^{2} d t+|\widehat{x}(T)|^{2}+|\widehat{X}(T)|^{2}\right\} .
\end{aligned}
$$

Then from the standard estimate of the mean-field BSDE part, we get

$$
\begin{aligned}
& E\left[\int_{0}^{T}\left(|\widehat{Y}(t)|^{2}+|\widehat{Z}(t)|^{2}\right) d t\right] \\
& \leq C_{1} \delta E\left\{\int_{0}^{T}|\widehat{\lambda}(t)|^{2} d t+|\widehat{x}(T)|^{2}\right\} \\
& \quad+C_{1}\left\{E \int_{0}^{T}|\widehat{X}(t)|^{2} d t+E|\widehat{X}(T)|^{2}\right\} .
\end{aligned}
$$

Here the constant $C_{1}$ depends on the Lipschitz constants, $\lambda_{1}, \beta_{1}, \mu_{1}, \alpha_{0}$, and $T$.

From the above two estimates and the standard estimate of $\widehat{X}(t)$, it follows that, for the sufficiently small $\delta>0$,

$$
\begin{aligned}
& E\left\{\int_{0}^{T}|\widehat{\Lambda}(t)|^{2} d t+|\widehat{X}(T)|^{2}\right\} \\
& \quad \leq \bar{C} \delta E\left\{\int_{0}^{T}|\widehat{\lambda}(t)| d t+|\widehat{x}(T)|^{2}\right\} .
\end{aligned}
$$

Here the constant $\bar{C}$ depends only on the Lipschitz constants, $\lambda_{1}, \beta_{1}, \mu_{1}, \alpha_{0}$, and $T$.

From above all, we now choose $\delta_{0}=1 / 2 \bar{C}$. Obviously, for every fixed $\delta \in\left[0, \delta_{0}\right]$, the mapping $I_{\alpha_{0}+\delta}$ is a contraction in the sense that

$$
\begin{aligned}
& E\left\{\int_{0}^{T}|\widehat{\Lambda}(t)|^{2} d t+|\widehat{X}(T)|^{2}\right\} \\
& \quad \leq \frac{1}{2} E\left\{\int_{0}^{T}|\widehat{\lambda}(t)| d t+|\widehat{x}(T)|^{2}\right\} .
\end{aligned}
$$

It means immediately that this mapping has a unique fixed point:

$$
\Lambda^{\alpha_{0}+\delta}=\left(X^{\alpha_{0}+\delta}, Y^{\alpha_{0}+\delta}, Z^{\alpha_{0}+\delta}\right),
$$

which is the solution of (15) for $\alpha=\alpha_{0}+\delta$.

Now we can give the proof of the existence of the solution of mean-field FBSDE (8).

Proof (continued). When $\alpha=0$, (15) has a unique solution. Then from Lemma 8 , there exists a positive constant $\delta_{0}$ 
depending on Lipschitz constants, $\beta_{1}, \mu_{1}, \lambda_{1}, \lambda_{2}$, and $T$, such that, for every $\delta \in\left[0, \delta_{0}\right]$, (15) for $\alpha=\delta$ has a unique solution. We can repeat this process $N$ times where $1 \leq$ $N \delta_{0} \leq 1+\delta_{0}$. It means that, in particular, mean-field FBSDE (15) for $\alpha=1$ has a unique solution; that is, (8) has a unique solution.

The proof is complete.

Example 9. We consider

$$
\begin{gathered}
d x(t)=E^{\prime}\left[-y^{\prime}(t)-2 y(t)\right] d t+E^{\prime}\left[-z^{\prime}(t)-2 z(t)\right] d B_{t}, \\
t \in[0, T], \\
-d y(t)=E^{\prime}\left[x^{\prime}(t)+2 x(t)\right] d t-z(t) d B_{t}, \quad t \in[0, T], \\
x(0)=1, \\
y(T)=E^{\prime}\left[x^{\prime}(T)+2 x(T)\right] .
\end{gathered}
$$

The above FBSDE satisfes (H3.1) and (H3.2), form Theorem 6, we know it has a unique solution.

Remark 10. The proof of Remark 7 is similar. Notice that (15) should be changed into the following form:

$$
\begin{gathered}
d x^{\alpha}(t)=\left[\alpha E^{\prime}\left[f\left(t, \chi^{\alpha}(t)\right)\right]+E^{\prime}[\phi(t)]\right] d t \\
+\left[\alpha E^{\prime}\left[\sigma\left(t, \chi^{\alpha}(t)\right)\right]+E^{\prime}[\psi(t)]\right] d B_{t}, \\
-d y^{\alpha}(t)=\left[-(1-\alpha) \beta_{1} G x^{\alpha}(t)\right. \\
\left.+\alpha E^{\prime}\left[g\left(t, \chi^{\alpha}(t)\right)\right]+E^{\prime}[\gamma(t)]\right] d t-z^{\alpha}(t) d B_{t}, \\
x^{\alpha}(0)=a, \\
y^{\alpha}(T)=\alpha E^{\prime}\left[\Phi\left(x^{\alpha}(T),\left(x^{\alpha}(T)\right)^{\prime}\right)\right] \\
-(1-\alpha) G x^{\alpha}(T)+\xi .
\end{gathered}
$$

Remark 11. When $\Phi$ does not depend on $x, \tilde{x}$, that is, $\Phi(x, \tilde{x})=\xi \in L^{2}\left(\Omega, \mathscr{F}_{T}, P\right)$ is given, for the existence and the uniqueness of the solution of mean-field FBSDE (8), the monotonicity assumption (H3.2) can be weakened as

$$
\langle A(t, \lambda, \tilde{\lambda})-A(t, \bar{\lambda}, \tilde{\lambda}), \lambda-\bar{\lambda}\rangle \leq-\beta_{1}|\widehat{x}|^{2}-\beta_{2}|\widehat{y}|^{2} ;
$$

similarly, (H3.3) can be weakened as

$$
\langle A(t, \lambda, \tilde{\lambda})-A(t, \bar{\lambda}, \tilde{\lambda}), \lambda-\bar{\lambda}\rangle \geq \beta_{1}|\widehat{x}|^{2}+\beta_{2}|\widehat{y}|^{2},
$$

where $\beta_{1}, \beta_{2}$, and $\mu_{1}$ are given nonnegative constants with $\beta_{1}-L_{A} \geq 0, \beta_{2}-L_{A} \geq 0$, and $\mu_{1}-L_{\Phi} \lambda_{1}>0$, where the equalities cannot be established at the same time; $L_{A}, L_{\Phi}$ are the Lipchitz constants of $A, \Phi$ with respect to $\tilde{\lambda}, \tilde{x}$, respectively; and $\lambda_{1}$ satisfies $|\widehat{G l}(T)| \leq \lambda_{1}|\widehat{l}(T)|$.

Lemma 12. When $\sigma$ does not depend on $z, z^{\prime}$, the meanfield FBSDE (8) also has a unique adapted solution, but the monotonicity (H3.2) should be weakened as (i) $\langle A(t, \lambda, \tilde{\lambda})-A(t, \bar{\lambda}, \tilde{\lambda}), \lambda-\bar{\lambda}\rangle \leq-\beta_{1}|\widehat{x}|^{2}$;

(ii) $\langle\Phi(x, \tilde{x})-\Phi(\bar{x}, \tilde{x}), G(x-\bar{x})\rangle \geq \mu_{1}|\widehat{x}|^{2}$;

similarly, (H3.3) can be weakened as

(i) $\langle A(t, \lambda, \tilde{\lambda})-A(t, \bar{\lambda}, \tilde{\lambda}), \lambda-\bar{\lambda}\rangle \geq \beta_{1}|\widehat{x}|^{2}$;

(ii) $\langle\Phi(x, \tilde{x})-\Phi(\bar{x}, \tilde{x}), G(x-\bar{x})\rangle \leq-\mu_{1}|\widehat{x}|^{2}$,

where $\beta_{1}$ and $\mu$ are given nonnegative constants. Moreover, one has $\beta_{1}>L_{A}+2 L_{A} C_{L_{g}, T}, \mu_{1}>L_{\Phi} \lambda_{1}+8 C_{L_{g}, T} L_{\Phi}^{2} L_{A}$, and $C_{L_{g}, T}=\exp \left\{\left(4 L_{g}+12 L_{g}^{2}+1\right) T\right\}$.

The proof of this lemma is similar to that of Theorem 6; we now only give the proof of the uniqueness.

Proof. Let $\lambda(t)=(x(t), y(t), z(t))$ and $\bar{\lambda}(t)=(\bar{x}(t), \bar{y}(t), \bar{z}(t))$ be two solutions of (8). We set $\hat{l}=l-\bar{l}$, where $l=x, y, z, \tilde{x}, \tilde{y}, \tilde{z}$, respectively. Applying Itô's formula to $\langle G \widehat{x}(s), \widehat{y}(s)\rangle$, we get

$$
\begin{gathered}
E\left\langle\left(E^{\prime}\left[\Phi\left(x(T),(x(T))^{\prime}\right)\right]-E^{\prime}\left[\Phi\left(\bar{x}(T),(\bar{x}(T))^{\prime}\right)\right]\right),\right. \\
G(x(T)-\bar{x}(T))\rangle \\
=E \int_{0}^{T}\left\langle\left( E^{\prime}\left[A\left(t, \lambda(t),(\lambda(t))^{\prime}\right)\right]\right.\right. \\
\left.-E^{\prime}\left[A\left(t, \bar{\lambda}(t),(\bar{\lambda}(t))^{\prime}\right)\right]\right), \\
\lambda(t)-\bar{\lambda}(t)\rangle d t .
\end{gathered}
$$

From (H3.2) the monotonicity assumptions of $\Phi$ and $A$, we get

$$
\begin{aligned}
\left(\mu_{1}-\right. & \left.L_{\Phi} \lambda_{1}\right) E\left[|\widehat{x}(T)|^{2}\right] \\
\leq & -\left(\beta_{1}-L_{A}\right) E \int_{0}^{T}|\widehat{x}(t)|^{2} d t \\
& +L_{A} E \int_{0}^{T}\left(|\widehat{y}(t)|^{2}+|\widehat{z}(t)|^{2}\right) d t
\end{aligned}
$$

Applying Itô's formula to $e^{\beta s}|\widehat{y}(s)|^{2}$, we get

$$
\begin{aligned}
d e^{\beta s}|\widehat{y}(s)|^{2} \\
=\beta e^{\beta s}|\widehat{y}(s)|^{2} d s+2 e^{\beta s} \widehat{y}(s) d \widehat{y}(s)+e^{\beta s}(d \widehat{y}(s))^{2} \\
=\beta e^{\beta s}|\widehat{y}(s)|^{2} d s-2 e^{\beta s} \widehat{y}(s) E^{\prime}[\widehat{g}] d s \\
\quad+2 e^{\beta s} \widehat{y}(s) \widehat{z}(s) d B_{s}+e^{\beta s}|\widehat{z}(s)|^{2} d s,
\end{aligned}
$$

where $\widehat{g}(s)=g\left(s, \lambda(s), \lambda^{\prime}(s)\right)-g\left(s, \bar{\lambda}(s),(\bar{\lambda}(s))^{\prime}\right)$. 
Then we get

$$
\begin{aligned}
E \int_{0}^{T} & \beta e^{\beta s}|\widehat{y}(s)|^{2} d s+E \int_{0}^{T} e^{\beta s}|\widehat{z}(s)|^{2} d s \\
= & E\left[e^{\beta T}|\widehat{y}(T)|^{2}\right]+E \int_{0}^{T} 2 e^{\beta s} \widehat{y}(s) E^{\prime}[\widehat{g}] d s \\
\leq & 4 e^{\beta T} L_{\Phi}^{2} E|\widehat{x}(T)|^{2}+E \int_{0}^{T} e^{\beta s}|\widehat{x}(s)|^{2} d s \\
& +\frac{1}{2} E \int_{0}^{T} e^{\beta s}|\widehat{z}(s)|^{2} d s \\
& +\left(4 L_{g}+12 L_{g}^{2}\right) E \int_{0}^{T} e^{\beta s}|\widehat{y}(s)|^{2} d s .
\end{aligned}
$$

Hence, we have

$$
\begin{aligned}
& E \int_{0}^{T}\left(\beta-4 L_{g}-12 L_{g}^{2}\right) e^{\beta s}|\widehat{y}(s)|^{2} d s \\
& \quad+\frac{1}{2} E \int_{0}^{T} e^{\beta s}|\widehat{z}(s)|^{2} d s \\
& \quad \leq 4 e^{\beta T} L_{\Phi}^{2} E|\widehat{x}(T)|^{2}+e^{\beta T} E \int_{0}^{T}|\widehat{x}(s)|^{2} d s .
\end{aligned}
$$

Thus, taking $\beta=4 L_{g}+12 L_{g}^{2}+1$, we get

$$
\begin{aligned}
& E \int_{0}^{T}\left(|\widehat{y}(s)|^{2}+|\widehat{z}(s)|^{2}\right) d s \\
& \quad \leq 8 C_{L_{g}, T} L_{\Phi}^{2} E|\widehat{x}(T)|^{2}+2 C_{L_{g}, T} E \int_{0}^{T}|\widehat{x}(s)|^{2} d s,
\end{aligned}
$$

where $C_{L_{g}, T}=\exp \left\{\left(4 L_{g}+12 L_{g}^{2}+1\right) T\right\}$.

Combining with (32), we have

$$
\begin{aligned}
& \left(\mu_{1}-L_{\Phi} \lambda_{1}-8 C_{L_{g}, T} L_{\Phi}^{2} L_{A}\right) E\left[|\widehat{x}(T)|^{2}\right] \\
& \quad \leq-\left(\beta_{1}-L_{A}-2 C_{L_{g}, T} L_{A}\right) E \int_{0}^{T}|\widehat{x}(t)|^{2} d t .
\end{aligned}
$$

When $\beta_{1}>L_{A}+2 L_{A} C_{L_{g}, T}, \mu_{1}>L_{\Phi} \lambda_{1}+8 C_{L_{g}, T} L_{\Phi}^{2} L_{A}$, we have $|\widehat{x}(t)|^{2}=0 d s d P$-a.e. In this case we have $\widehat{x}(t)=0, P$-a.s., for all $t \in[0, T]$. Thus, $\Phi\left(x(T),(x(T))^{\prime}\right)=\Phi\left(\bar{x}(T),(\bar{x}(T))^{\prime}\right), \bar{P}_{-}$ a.s. Therefore, from Lemma 1 it follows that $y(t)=\bar{y}(t), P$-a.s. and $z(t)=\bar{z}(t), P$-a.s.

\section{Continuity Property on the Parameters}

In this section we will discuss the continuity of the solution of (8) depending on parameters. We consider the following mean-field FBSDEs with coefficients $\left(f_{\alpha}, \sigma_{\alpha}, g_{\alpha}, \Phi_{\alpha}\right), \alpha \in \mathbb{R}$ :

$$
\begin{gathered}
d x^{\alpha}(t)=E^{\prime}\left[f_{\alpha}\left(t, \chi^{\alpha}(t)\right)\right] d t+E^{\prime}\left[\sigma_{\alpha}\left(t, \chi^{\alpha}(t)\right)\right] d B_{t}, \\
-d y^{\alpha}(t)=E^{\prime}\left[g_{\alpha}\left(t, \chi^{\alpha}(t)\right)\right] d t-z^{\alpha}(t) d B_{t}, \\
x^{\alpha}(0)=a, \\
y^{\alpha}(T)=E^{\prime}\left[\Phi_{\alpha}\left(x^{\alpha}(T),\left(x^{\alpha}(T)\right)^{\prime}\right)\right],
\end{gathered}
$$

where $\chi^{\alpha}(t)=\left(x^{\alpha}(t), y^{\alpha}(t), z^{\alpha}(t),\left(x^{\alpha}(t)\right)^{\prime},\left(y^{\alpha}(t)\right)^{\prime},\left(z^{\alpha}(t)\right)^{\prime}\right)$ and $f_{\alpha}, \sigma_{\alpha}, g_{\alpha}, \Phi_{\alpha}, \alpha \in \mathbb{R}$, satisfy (H3.1) and (H3.2) for each $\alpha \in \mathbb{R}$. Then, from Theorem 6 we know that mean-field $\operatorname{FBSDE}(38)$ has a unique solution $\left(x^{\alpha}, y^{\alpha}, z^{\alpha}\right)$ for each $\alpha \in \mathbb{R}$.

Let us give some more assumptions.

(H4.1)

(i) The coefficients $\left(f_{\alpha}, \sigma_{\alpha}, g_{\alpha}, \Phi_{\alpha}\right), \alpha \in \mathbb{R}$, are uniformly Lipschitz to $(x, y, z, \tilde{x}, \tilde{y}, \widetilde{z})$;

(ii) the mappings $\alpha \mapsto\left(f_{\alpha}, \sigma_{\alpha}, g_{\alpha}, \Phi_{\alpha}\right), \alpha \in \mathbb{R}$, are continuous, respectively.

Then we have the following continuity property.

Theorem 13. Let the coefficients $\left(f_{\alpha}, \sigma_{\alpha}, g_{\alpha}, \Phi_{\alpha}\right), \alpha \in \mathbb{R}$, satisfy (H3.1), (H3.2), and (H4.1), and the associated solution of mean-field FBSDE (38) is denoted by $\left(x^{\alpha}, y^{\alpha}, z^{\alpha}\right)$. Then, the mappings

$$
\begin{aligned}
\alpha \longmapsto & \left(x^{\alpha}, y^{\alpha}, z^{\alpha}, x^{\alpha}(T)\right): \mathbb{R} \longmapsto M_{\mathbb{F}}^{2} \\
& \times\left(0, T ; \mathbb{R}^{n} \times \mathbb{R}^{m} \times \mathbb{R}^{m \times d}\right) \\
& \times L^{2}\left(\Omega, \mathscr{F}_{T}, \mathbf{P} ; \mathbb{R}^{n}\right)
\end{aligned}
$$

are continuous.

Proof. For simplicity of notations, we only prove the continuity of the solutions $\left(x^{\alpha}, y^{\alpha}, z^{\alpha}, x^{\alpha}(T)\right)$ of mean-field FBSDE (38) at $\alpha=0$. We want to prove that $\left(x^{\alpha}, y^{\alpha}, z^{\alpha}, x^{\alpha}(T)\right)$ converges to $\left(x^{0}, y^{0}, z^{0}, x^{0}(T)\right)$ in $M_{\mathbb{F}}^{2}\left(0, T ; \mathbb{R}^{n} \times \mathbb{R}^{m} \times\right.$ $\left.\mathbb{R}^{m \times d}\right) \times L^{2}\left(\Omega, \mathscr{F}_{T}, \mathbf{P} ; \mathbb{R}^{n}\right)$ as $\alpha$ tends to 0 . We set $\lambda^{\alpha}(t)=$ $\left(x^{\alpha}(t), y^{\alpha}(t), z^{\alpha}(t)\right)$ and $\hat{\lambda}(t)=\left(\lambda^{\alpha}(t)-\lambda^{0}(t)\right)=(\widehat{x}(t)$, $\widehat{y}(t), \widehat{z}(t))=\left(x^{\alpha}(t)-x^{0}(t), y^{\alpha}(t)-y^{0}(t), z^{\alpha}(t)-z^{0}(t)\right)$; then from (38) we know that

$$
\begin{gathered}
d \widehat{x}(t)=E^{\prime}\left[f_{\alpha}\left(t, \lambda^{\alpha}(t),\left(\lambda^{\alpha}(t)\right)^{\prime}\right)\right. \\
\left.-f_{0}\left(t, \lambda^{0}(t),\left(\lambda^{0}(t)\right)^{\prime}\right)\right] d t \\
+E^{\prime}\left[\sigma_{\alpha}\left(t, \lambda^{\alpha}(t),\left(\lambda^{\alpha}(t)\right)^{\prime}\right)\right. \\
\left.-\sigma_{0}\left(t, \lambda^{0}(t),\left(\lambda^{0}(t)\right)^{\prime}\right)\right] d B_{t}, \\
-d \widehat{y}(t)=E^{\prime}\left[g_{\alpha}\left(t, \lambda^{\alpha}(t),\left(\lambda^{\alpha}(t)\right)^{\prime}\right)\right. \\
\left.-g_{0}\left(t, \lambda^{0}(t),\left(\lambda^{0}(t)\right)^{\prime}\right)\right] d t \\
-\widehat{z}(t) d B_{t}, \\
\left.\widehat{x}(0)=0, \quad-\Phi_{0}\left(x^{0}(T),\left(x^{0}(T)\right)^{\prime}\right)\right] . \\
\widehat{y}(T)=E^{\prime}\left[\Phi_{\alpha}\left(x^{\alpha}(T),\left(x^{\alpha}(T)\right)^{\prime}\right)\right.
\end{gathered}
$$


From assumptions (H3.1), (H3.2), and (H4.1) and standard estimates of $\widehat{x}(t)$ and $(\widehat{y}(t), \widehat{z}(t))$, we get

$$
\begin{aligned}
& \sup _{0 \leq t \leq T} E|\widehat{x}(t)|^{2} \\
& \leq C_{1} E \int_{0}^{T}\left(|\widehat{y}(t)|^{2}+|\widehat{z}(t)|^{2}\right) d t \\
& +C_{1} \bar{E} \int_{0}^{T}\left[|\widehat{f}(t)|^{2}+|\widehat{\sigma}(t)|^{2}\right] d t \\
& E \int_{0}^{T}\left(|\widehat{y}(t)|^{2}+|\widehat{z}(t)|^{2}\right) d t \\
& \leq C_{1}\left\{E \int_{0}^{T}|\widehat{x}(t)|^{2} d t+E|\widehat{x}(T)|^{2}\right. \\
& \left.+\bar{E} \int_{0}^{T}|\widehat{g}(t)|^{2} d t+\bar{E}\left[|\widehat{\Phi}(T)|^{2}\right]\right\},
\end{aligned}
$$

where $C_{1}$ depends on the Lipchitz constants and $T$, where

$$
\begin{aligned}
& \widehat{f}(t)=f_{\alpha}\left(t, \lambda^{0}(t),\left(\lambda^{0}(t)\right)^{\prime}\right)-f_{0}\left(t, \lambda^{0}(t),\left(\lambda^{0}(t)\right)^{\prime}\right), \\
& \widehat{\sigma}(t)=\sigma_{\alpha}\left(t, \lambda^{0}(t),\left(\lambda^{0}(t)\right)^{\prime}\right)-\sigma_{0}\left(t, \lambda^{0}(t),\left(\lambda^{0}(t)\right)^{\prime}\right), \\
& \widehat{g}(t)=-g_{\alpha}\left(t, \lambda^{0}(t),\left(\lambda^{0}(t)\right)^{\prime}\right)+g_{0}\left(t, \lambda^{0}(t),\left(\lambda^{0}(t)\right)^{\prime}\right), \\
& \widehat{\Phi}(T)=\Phi_{\alpha}\left(x^{0}(T),\left(x^{0}(T)\right)^{\prime}\right)-\Phi_{0}\left(x^{0}(T),\left(x^{0}(T)\right)^{\prime}\right) .
\end{aligned}
$$

Applying Itô's formula to $\langle G \widehat{x}(t), \widehat{y}(t)\rangle$, it yields

$$
\begin{gathered}
E\left\langleE ^ { \prime } \left[\Phi_{\alpha}\left(x^{\alpha}(T),\left(x^{\alpha}(T)\right)^{\prime}\right)\right.\right. \\
\left.\left.-\Phi_{\alpha}\left(x^{0}(T),\left(x^{0}(T)\right)^{\prime}\right)\right], G \hat{x}(T)\right\rangle \\
+E\left\langleE ^ { \prime } \left[\Phi_{\alpha}\left(x^{0}(T),\left(x^{0}(T)\right)^{\prime}\right)\right.\right. \\
\left.\left.-\Phi_{0}\left(x^{0}(T),\left(x^{0}(T)\right)^{\prime}\right)\right], G \widehat{x}(T)\right\rangle \\
=E \int_{0}^{T} E^{\prime}\left\langle A_{\alpha}\left(t, \lambda^{\alpha}(t),\left(\lambda^{\alpha}(t)\right)^{\prime}\right)\right. \\
\left.-A_{\alpha}\left(t, \lambda^{0}(t),\left(\lambda^{0}(t)\right)^{\prime}\right), \widehat{\lambda}(t)\right\rangle d t \\
+E \int_{0}^{T} E^{\prime}\left[\langle G \widehat{x}(t), \widehat{g}(t)\rangle+\left\langle G^{T} \hat{y}(t), \widehat{f}(t)\right\rangle\right. \\
\left.+\left\langle G^{T} \widehat{z}(t), \widehat{\sigma}(t)\right\rangle\right] d t,
\end{gathered}
$$

where

$$
A_{\alpha}\left(t, \lambda, \lambda^{\prime}\right)=\left(\begin{array}{c}
-G^{T} g_{\alpha} \\
G f_{\alpha} \\
G \sigma_{\alpha}
\end{array}\right)\left(t, \lambda, \lambda^{\prime}\right)
$$

Then, we have

$$
\begin{gathered}
\left(\beta_{1}-L_{A}\right) E \int_{0}^{T}|\widehat{x}(t)|^{2} d t+\left(\mu_{1}-L_{\Phi} \lambda_{1}\right) E|\widehat{x}(T)|^{2} \\
+\left(\beta_{2}-L_{A}\right) E \\
\quad \times \int_{0}^{T}\left(|\widehat{y}(t)|^{2}+|\widehat{z}(t)|^{2}\right) d t \\
\leq C_{2} E\left[E^{\prime}|\widehat{\Phi}(T)|^{2}\right. \\
\left.\quad+\int_{0}^{T} E^{\prime}\left(|\widehat{f}(t)|^{2}+|\widehat{g}(t)|^{2}+|\widehat{\sigma}(t)|^{2}\right) d t\right] \\
+\delta\left[E|\widehat{x}(T)|^{2}+E \int_{0}^{T}\left(|\widehat{x}(t)|^{2}+|\widehat{y}(t)|^{2}+|\widehat{z}(t)|^{2}\right) d t\right]
\end{gathered}
$$

From (H3.2) we know if $\beta_{1}-L_{A}>0, \mu_{1}-L_{\Phi} \lambda_{1}>0$, then $\beta_{2}-L_{A} \geq 0$. Then, from (46) we have

$$
\begin{aligned}
& \left(\beta_{1}-L_{A}\right) E \int_{0}^{T}|\widehat{x}(t)|^{2} d t+\left(\mu_{1}-L_{\Phi} \lambda_{1}\right) E|\widehat{x}(T)|^{2} \\
& \leq C_{2} E\left[E^{\prime}|\widehat{\Phi}(T)|^{2}\right. \\
& \left.+\int_{0}^{T} E^{\prime}\left(|\widehat{f}(t)|^{2}+|\widehat{g}(t)|^{2}+|\widehat{\sigma}(t)|^{2}\right) d t\right] \\
& +\delta\left[E|\widehat{x}(T)|^{2}+E \int_{0}^{T}\left(|\widehat{x}(t)|^{2}+|\widehat{y}(t)|^{2}+|\widehat{z}(t)|^{2}\right) d t\right]
\end{aligned}
$$

With the help of (42) and (47) we can take sufficiently small $\delta$ such that

$$
\begin{aligned}
& E|\widehat{x}(T)|^{2}+E \int_{0}^{T}|\widehat{\lambda}(t)|^{2} d t \\
& \leq C \bar{E}\left[|\widehat{\Phi}(T)|^{2}\right. \\
&\left.+\int_{0}^{T}\left(|\widehat{f}(t)|^{2}+|\widehat{g}(t)|^{2}+|\widehat{\sigma}(t)|^{2}\right) d t\right]
\end{aligned}
$$

where the constant $C$ only depends on $C_{1}, C_{2}, \beta_{1}, \mu_{1}, L_{A}, L_{\Phi}$. 
Similarly, if $\beta_{1}-L_{A}=0, \mu_{1}-L_{\Phi} \lambda_{1}>0$, then $\beta_{2}-L_{A}>0$. Then, from (46) we have

$$
\begin{aligned}
& \left(\beta_{2}-L_{A}\right) E \int_{0}^{T}\left(|\widehat{y}(t)|^{2}+|\widehat{z}(t)|^{2}\right) d t \\
& \leq C_{2} E\left[E^{\prime}|\widehat{\Phi}(T)|^{2}\right. \\
& \left.\quad+\int_{0}^{T} E^{\prime}\left(|\widehat{f}(t)|^{2}+|\widehat{g}(t)|^{2}+|\widehat{\sigma}(t)|^{2}\right) d t\right] \\
& +\delta\left[E|\widehat{x}(T)|^{2}\right. \\
& \left.\quad+E \int_{0}^{T}\left(|\widehat{x}(t)|^{2}+|\widehat{y}(t)|^{2}+|\widehat{z}(t)|^{2}\right) d t\right] .
\end{aligned}
$$

With the help of (41) and (49) we can take sufficiently small $\delta$ such that

$$
\begin{aligned}
& E|\widehat{x}(T)|^{2}+E \int_{0}^{T}|\widehat{\lambda}(t)|^{2} d t \\
& \quad \leq C \bar{E}\left[|\widehat{\Phi}(T)|^{2}+\int_{0}^{T}\left(|\widehat{f}(t)|^{2}+|\widehat{g}(t)|^{2}+|\widehat{\sigma}(t)|^{2}\right) d t\right],
\end{aligned}
$$

where the constant $C$ only depends on $C_{1}, C_{2}, \beta_{2}, L_{A}, L_{\Phi}$.

Hence, we have that $\left(x^{\alpha}, y^{\alpha}, z^{\alpha}, x^{\alpha}(T)\right)$ converges to $\left(x^{0}\right.$, $\left.y^{0}, z^{0}, x^{0}(T)\right)$ in $M_{\mathbb{F}}^{2}\left(0, T ; \mathbb{R}^{n} \times \mathbb{R}^{m} \times \mathbb{R}^{m \times d}\right) \times L^{2}\left(\Omega, \mathscr{F}_{T}, \mathbf{P} ; \mathbb{R}^{n}\right)$ as $\alpha$ tends to 0 .

Now we will give an example to explain that (H3.2) (or (H3.3)) is necessary; that is, if the coefficients do not satisfy (H3.2) (or (H3.3)), then (8) may not have a solution. We take $m=n=d=1$ here. We consider

$$
\begin{gathered}
d x(t)=E[y(t)] d t+d B_{t}, \quad t \in\left[0, \frac{3}{4} \pi\right], \\
-d y(t)=E[x(t)] d t-z(t) d B_{t}, \quad t \in\left[0, \frac{3}{4} \pi\right], \\
x(0)=1, \quad y\left(\frac{3}{4} \pi\right)=-E\left[x\left(\frac{3}{4} \pi\right)\right], \quad t \in\left[0, \frac{3}{4} \pi\right] .
\end{gathered}
$$

It is easy to check that this equation does not satisfy (H3.2) (or (H3.3)); we point out that, there is also no adapted solution. In fact, if $(x, y, z)_{0 \leq t \leq(3 / 4) \pi}$ is the solution of mean-field FBSDE (51), then $(E[x(t)], E[y(t)])$ is the solution of the following ordinary differential equation:

$$
\begin{gathered}
\dot{X}=Y, \quad \dot{Y}=-X, \\
X(0)=1, \quad Y\left(\frac{3}{4} \pi\right)=-X\left(\frac{3}{4} \pi\right), \quad t \in\left[0, \frac{3}{4} \pi\right] .
\end{gathered}
$$

But we know this ODE has no solution; therefore, there is no adapted solution of (51).

\section{Maximum Principle for the Controlled Mean-Field FBSDEs}

We consider the following controlled mean-field fully coupled forward-backward SDEs:

$$
\begin{gathered}
d x(t)=E^{\prime}[f(t, \chi(t), v(t))] d t+E^{\prime}[\sigma(t, \chi(t), v(t))] d B_{t}, \\
-d y(t)=E^{\prime}[g(t, \chi(t), v(t))] d t-z(t) d B_{t}, \\
x(0)=a, \quad y(T)=E^{\prime}\left[\Phi\left(x(T),(x(T))^{\prime}\right)\right],
\end{gathered}
$$

where $\chi(t)=\left(x(t), y(t), z(t),(x(t))^{\prime},(y(t))^{\prime},(z(t))^{\prime}\right),(x, y, z)$ takes value in $\mathbb{R}^{n} \times \mathbb{R}^{m} \times \mathbb{R}^{m \times d}$. Let $U$ be a nonempty convex subset of $\mathbb{R}^{k}$

$$
\begin{aligned}
& \mathcal{U}_{\mathrm{ad}} \\
& =\left\{v(\cdot) \in M_{\overline{\mathbb{F}}}^{2}\left(0, T ; \mathbb{R}^{k}\right) \mid v(t) \in U, 0 \leq t \leq T, \bar{P} \text {-a.s. }\right\} .
\end{aligned}
$$

An element $v$ of $\mathscr{U}_{\mathrm{ad}}$ is called an admissible control. We define the following cost functional:

$$
\begin{aligned}
J(v(\cdot))=E\left[\int_{0}^{T} E^{\prime}[L(\chi(t), v(t))] d t\right. \\
\\
\left.\quad+E^{\prime}\left[\varphi\left(x(T),(x(T))^{\prime}\right)\right]+h(y(0))\right],
\end{aligned}
$$

where

$$
\begin{aligned}
f: & {[0, T] \times \mathbb{R}^{n} \times \mathbb{R}^{m} \times \mathbb{R}^{m \times d} } \\
& \times \mathbb{R}^{n} \times \mathbb{R}^{m} \times \mathbb{R}^{m \times d} \times \mathbb{R}^{k} \longrightarrow \mathbb{R}^{n}, \\
\sigma: & {[0, T] \times \mathbb{R}^{n} \times \mathbb{R}^{m} \times \mathbb{R}^{m \times d} } \\
& \times \mathbb{R}^{n} \times \mathbb{R}^{m} \times \mathbb{R}^{m \times d} \times \mathbb{R}^{k} \longrightarrow \mathbb{R}^{n \times d}, \\
g: & {[0, T] \times \mathbb{R}^{n} \times \mathbb{R}^{m} \times \mathbb{R}^{m \times d} } \\
& \times \mathbb{R}^{n} \times \mathbb{R}^{m} \times \mathbb{R}^{m \times d} \times \mathbb{R}^{k} \longrightarrow \mathbb{R}^{m}, \\
L & :[0, T] \times \mathbb{R}^{n} \times \mathbb{R}^{m} \times \mathbb{R}^{m \times d} \\
& \times \mathbb{R}^{n} \times \mathbb{R}^{m} \times \mathbb{R}^{m \times d} \times \mathbb{R}^{k} \longrightarrow \mathbb{R}, \\
\Phi & : \mathbb{R}^{n} \times \mathbb{R}^{n} \longrightarrow \mathbb{R}^{m}, \\
\varphi & : \mathbb{R}^{n} \times \mathbb{R}^{n} \longrightarrow \mathbb{R}, \quad h: \mathbb{R}^{m} \longrightarrow \mathbb{R} .
\end{aligned}
$$

The optimal control problem is to minimize the cost functional $J(v(\cdot))$ over all admissible controls. An admissible control $u(\cdot)$ is called an optimal control if the cost functional $J(v(\cdot))$ attains the minimum at $u(\cdot)$. Equation (53) is called the state equation; the solution $(x(\cdot), y(\cdot), z(\cdot))$ corresponding to $u(\cdot)$ is called the optimal trajectory. 
We assume the following.

(H5.1)

(i) $f, g, \sigma, L, \Phi, \varphi$ and $h$ are continuously differentiable to $(x, y, z, \tilde{x}, \tilde{y}, \widetilde{z}, v)$;

(ii) the derivatives of $f, g, \sigma, \Phi$ are bounded;

(iii) the derivatives of $L$ are bounded by $C(1+|x|+|y|+$ $|z|+|\widetilde{x}|+|\widetilde{y}|+|\widetilde{z}|+|v|)$

(iv) the derivatives of $\varphi$ and $h$ are bounded by $C(1+|x|+$ $|\widetilde{x}|)$ and $C(1+|y|)$, respectively;

(v) for any given admissible control $v(\cdot)$, the coefficients satisfy (H3.1) and (H3.2).

Let $u(\cdot)$ be an optimal control and let $(x(\cdot), y(\cdot), z(\cdot))$ be the corresponding optimal trajectory. Let $v(\cdot)$ be such that $u(\cdot)+v(\cdot) \in \mathcal{U}_{\mathrm{ad}}$. Since $U$ is convex, then for any $0 \leq \rho \leq$ $1, u_{\rho}(\cdot)=u(\cdot)+\rho v(\cdot)$ is also in $\mathcal{U}_{\mathrm{ad}}$.

We introduce the following linear mean-field FBSDE:

$$
\begin{aligned}
d x^{1}(t) & \\
=\left\{E^{\prime}\right. & {\left[f_{x}(\theta(t))\right] x^{1}(t)+E^{\prime}\left[f_{y}(\theta(t))\right] y^{1}(t) } \\
& +E^{\prime}\left[f_{z}(\theta(t))\right] z^{1}(t)+E^{\prime}\left[f_{v}(\theta(t)) v(t)\right] \\
& +E^{\prime}\left[f_{\tilde{x}}(\theta(t))\left(x^{1}(t)\right)^{\prime}\right]+E^{\prime}\left[f_{\tilde{y}}(\theta(t))\left(y^{1}(t)\right)^{\prime}\right] \\
& \left.+E^{\prime}\left[f_{\tilde{z}}(\theta(t))\left(z^{1}(t)\right)^{\prime}\right]\right\} d t \\
+ & \left\{E^{\prime}\left[\sigma_{x}(\theta(t))\right] x^{1}(t)+E^{\prime}\left[\sigma_{y}(\theta(t))\right] y^{1}(t)\right. \\
& +E^{\prime}\left[\sigma_{z}(\theta(t))\right] z^{1}(t)+E^{\prime}\left[\sigma_{v}(\theta(t)) v(t)\right] \\
& +E^{\prime}\left[\sigma_{\tilde{x}}(\theta(t))\left(x^{1}(t)\right)^{\prime}\right]+E^{\prime}\left[\sigma_{\widetilde{y}}(\theta(t))\left(y^{1}(t)\right)^{\prime}\right] \\
& \left.+E^{\prime}\left[\sigma_{\widetilde{z}}(\theta(t))\left(z^{1}(t)\right)^{\prime}\right]\right\} d B_{t},
\end{aligned}
$$$$
-d y^{1}(t)
$$$$
=\left\{E^{\prime}\left[g_{x}(\theta(t))\right] x^{1}(t)+E^{\prime}\left[g_{y}(\theta(t))\right] y^{1}(t)\right.
$$$$
+E^{\prime}\left[g_{z}(\theta(t))\right] z^{1}(t)
$$$$
+E^{\prime}\left[g_{v}(\theta(t)) v(t)\right]+E^{\prime}\left[g_{\widetilde{x}}(\theta(t))\left(x^{1}(t)\right)^{\prime}\right]
$$$$
+E^{\prime}\left[g_{\tilde{y}}(\theta(t))\left(y^{1}(t)\right)^{\prime}\right]
$$$$
\left.+E^{\prime}\left[g_{\tilde{z}}(\theta(t))\left(z^{1}(t)\right)^{\prime}\right]\right\} d t
$$$$
-z^{1}(t) d B_{t}
$$

$x^{1}(0)=0$,

$$
y^{1}(T)=E^{\prime}\left[\Phi_{x}\left(x(T),(x(T))^{\prime}\right) x^{1}(T),\right.
$$

$$
\left.+\Phi_{\widetilde{x}}\left(x(T),(x(T))^{\prime}\right)\left(x^{1}(T)\right)^{\prime}\right],
$$

where $\theta(t)=\left(t, x(t), y(t), z(t),(x(t))^{\prime},(y(t))^{\prime},(z(t))^{\prime}, u(t)\right)$.

Remark 14. When $l=f, g, \sigma, L$, respectively, $l_{x}$ is the partial derivative of $l(t, x, y, z, \tilde{x}, \tilde{y}, \tilde{z}, v)$ with respect to $x ; l_{\tilde{x}}$ is the partial derivative of $l(t, x, y, z, \tilde{x}, \tilde{y}, \widetilde{z}, v)$ with respect to $\tilde{x}$, similar to $l_{y}, l_{\tilde{\gamma}}, l_{z}, l_{\widetilde{z}}, l_{v}$.

From (H5.1), it is easy to verify that (57) satisfies (H3.1) and (H3.2); then there exists a unique solution $\left(x^{1}, y^{1}, z^{1}\right)$ of mean-field FBSDE (57). Equation (57) is called the variational equation.

We denote by $\left(x_{\rho}(\cdot), y_{\rho}(\cdot), z_{\rho}(\cdot)\right)$ the trajectory corresponding to $u_{\rho}$. Then we have the following convergence result.

Lemma 15. One assumes (H5.1) holds. Then, $\lim _{\rho \rightarrow 0}\left(\left(x_{\rho}(t)-\right.\right.$ $x(t)) / \rho)=x^{1}(t), \quad \lim _{\rho \rightarrow 0}\left(\left(y_{\rho}(t)-y(t)\right) / \rho\right)=y^{1}(t)$, and $\lim _{\rho \rightarrow 0}\left(\left(z_{\rho}(t)-z(t)\right) / \rho\right)=z^{1}(t)$, in $M_{\mathbb{F}}^{2}(0, T)$.

Proof. Let $\widehat{x}(t)=x_{\rho}(t)-x(t), \hat{y}(t)=y_{\rho}(t)-y(t), \widehat{z}(t)=$ $z_{\rho}(t)-z(t)$. Then

$$
\begin{gathered}
d \widehat{x}(t)=E^{\prime}\left[f\left(\chi_{\rho}(t), u(t)+\rho v(t)\right)-f(\chi(t), u(t))\right] d t \\
+E^{\prime}\left[\sigma\left(\chi_{\rho}(t), u(t)+\rho v(t)\right)\right. \\
-\sigma(\chi(t), u(t))] d B_{t}, \\
-d \hat{y}(t)=E^{\prime}\left[g\left(\chi_{\rho}(t), u(t)+\rho v(t)\right)-g(\chi(t), u(t))\right] d t \\
-\widehat{z}(t) d B_{t}, \\
\widehat{x}(0)=0, \quad \\
\hat{y}(T)=E^{\prime}\left[\Phi\left(x_{\rho}(T),\left(x_{\rho}(T)\right)^{\prime}\right)\right. \\
\left.-\Phi\left(x(T),(x(T))^{\prime}\right)\right],
\end{gathered}
$$

$\chi_{\rho}(t)=\left(t, x_{\rho}(t), y_{\rho}(t), \quad z_{\rho}(t),\left(x_{\rho}(t)\right)^{\prime},\left(y_{\rho}(t)\right)^{\prime}\right.$, $\left.\left(z_{\rho}(t)\right)^{\prime}\right)$ and $\chi(t)=\left(t, x(t), y(t), z(t),(x(t))^{\prime},(y(t))^{\prime},(z(t))^{\prime}\right)$. From Theorem 13 , it is easy to know that $(\widehat{x}(\cdot), \widehat{y}(\cdot), \widehat{z}(\cdot))$ converges to 0 in $M_{\mathbb{F}}^{2}(0, T)$ as $\rho$ tends to 0 . Now, we define

$$
\begin{aligned}
& \Delta x(t)=\frac{x_{\rho}(t)-x(t)}{\rho}, \quad \Delta y(t)=\frac{y_{\rho}(t)-y(t)}{\rho}, \\
& \Delta z(t)=\frac{z_{\rho}(t)-z(t)}{\rho}, \\
& \lambda(t)=(x(t), y(t), z(t)), \\
& \lambda_{\rho}(t)=\left(x_{\rho}(t), y_{\rho}(t), z_{\rho}(t)\right) .
\end{aligned}
$$


Then,

$$
\begin{gathered}
d \Delta x(t)=\frac{1}{\rho} E^{\prime}\left[f\left(t, \lambda_{\rho}(t),\left(\lambda_{\rho}(t)\right)^{\prime}, u(t)+\rho v(t)\right)\right. \\
\left.-f\left(t, \lambda(t),(\lambda(t))^{\prime}, u(t)\right)\right] d t \\
+\frac{1}{\rho} E^{\prime}\left[\sigma\left(t, \lambda_{\rho}(t),\left(\lambda_{\rho}(t)\right)^{\prime}, u(t)+\rho v(t)\right)\right. \\
\left.-\sigma\left(t, \lambda(t),(\lambda(t))^{\prime}, u(t)\right)\right] d B_{t}, \\
-d \Delta y(t) \\
=\frac{1}{\rho} E^{\prime}\left[g\left(t, \lambda_{\rho}(t),\left(\lambda_{\rho}(t)\right)^{\prime}, u(t)+\rho v(t)\right)\right. \\
\left.-g\left(t, \lambda(t),(\lambda(t))^{\prime}, u(t)\right)\right] d t \\
-\Delta z(t) d B_{t}, \\
\left.\Delta x(0)=0, \quad-\Phi\left(x(T),(x(T))^{\prime}\right)\right] . \\
\Delta y(T)=\frac{1}{\rho} E^{\prime}\left[\Phi\left(x_{\rho}(T),\left(x_{\rho}(T)\right)^{\prime}\right)\right.
\end{gathered}
$$

The above Equation(60) can be rewritten as the following:

$$
\begin{gathered}
d \Delta x(t)=E^{\prime}[\bar{f}(\Delta \chi(t), v(t))] d t \\
+E^{\prime}[\bar{\sigma}(\Delta \chi(t), v(t))] d B_{t}, \\
-d \Delta y(t)=E^{\prime}[\bar{g}(\Delta \chi(t), v(t))] d t-\Delta z(t) d B_{t}, \\
\begin{aligned}
\Delta x(0)=0, \\
\Delta y(T)=E^{\prime}\left[\bar{K}^{l}(T) \Delta x(T)+\bar{M}^{l}(T)(\Delta x(T))^{\prime}\right],
\end{aligned}
\end{gathered}
$$

where

$$
\Delta \chi(t)
$$$$
=\left(t, \Delta x(t), \Delta y(t), \Delta z(t),(\Delta x(t))^{\prime},(\Delta y(t))^{\prime},(\Delta z(t))^{\prime}\right),
$$

$$
\bar{l}(t, x, y, z, \tilde{x}, \tilde{y}, \widetilde{z}, v)
$$

$$
\begin{aligned}
= & A^{l}(t) x+B^{l}(t) y+C^{l}(t) z \\
& +D^{l}(t) \tilde{x}+E^{l}(t) \tilde{y}+F^{l}(t) \tilde{z}+G^{l}(t) v,
\end{aligned}
$$

where $l=f, g, \sigma$, respectively, and

$A(t)$

$$
\begin{aligned}
= & \frac{1}{x_{\rho}(t)-x(t)} \\
& \cdot\left[l\left(t, x_{\rho}(t), y_{\rho}(t), z_{\rho}(t),\left(\lambda_{\rho}(t)\right)^{\prime}, u(t)+\rho v(t)\right)\right. \\
& \left.-l\left(t, x(t), y_{\rho}(t), z_{\rho}(t),\left(\lambda_{\rho}(t)\right)^{\prime}, u(t)+\rho v(t)\right)\right],
\end{aligned}
$$

$B(t)$

$$
\begin{aligned}
= & \frac{1}{y_{\rho}(t)-y(t)} \\
& \cdot\left[l\left(t, x(t), y_{\rho}(t), z_{\rho}(t),\left(\lambda_{\rho}(t)\right)^{\prime}, u(t)+\rho v(t)\right)\right. \\
& \left.-l\left(t, x(t), y(t), z_{\rho}(t),\left(\lambda_{\rho}(t)\right)^{\prime}, u(t)+\rho v(t)\right)\right],
\end{aligned}
$$

$C(t)$

$$
\begin{aligned}
= & \frac{1}{z_{\rho}(t)-z(t)} \\
& \cdot\left[l\left(t, x(t), y(t), z_{\rho}(t),\left(\lambda_{\rho}(t)\right)^{\prime}, u(t)+\rho v(t)\right)\right.
\end{aligned}
$$

$$
\left.-l\left(t, x(t), y(t), z(t),\left(\lambda_{\rho}(t)\right)^{\prime}, u(t)+\rho v(t)\right)\right],
$$

$D(t)$

$$
\begin{aligned}
= & \frac{1}{\left(x_{\rho}(t)\right)^{\prime}-(x(t))^{\prime}} \\
\cdot & {\left[l\left(t, \lambda(t),\left(x_{\rho}(t)\right)^{\prime},\left(y_{\rho}(t)\right)^{\prime},\left(z_{\rho}(t)\right)^{\prime}, u(t)+\rho v(t)\right)\right.} \\
& \left.-l\left(t, \lambda(t),(x(t))^{\prime},\left(y_{\rho}(t)\right)^{\prime},\left(z_{\rho}(t)\right)^{\prime}, u(t)+\rho v(t)\right)\right], \\
E & (t) \quad \\
= & \frac{1}{\left(y_{\rho}(t)\right)^{\prime}-(y(t))^{\prime}} \\
& \cdot\left[l\left(t, \lambda(t),(x(t))^{\prime},\left(y_{\rho}(t)\right)^{\prime},\left(z_{\rho}(t)\right)^{\prime}, u(t)+\rho v(t)\right)\right. \\
& \left.-l\left(t, \lambda(t),(x(t))^{\prime},(y(t))^{\prime},\left(z_{\rho}(t)\right)^{\prime}, u(t)+\rho v(t)\right)\right],
\end{aligned}
$$

$F(t)$

$$
\begin{aligned}
= & \frac{1}{\left(z_{\rho}(t)\right)^{\prime}-(z(t))^{\prime}} \\
& \cdot\left[l\left(t, \lambda(t),(x(t))^{\prime},(y(t))^{\prime},\left(z_{\rho}(t)\right)^{\prime}, u(t)+\rho v(t)\right)\right. \\
& \left.\quad l\left(t, \lambda(t),(x(t))^{\prime},(y(t))^{\prime},(z(t))^{\prime}, u(t)+\rho v(t)\right)\right],
\end{aligned}
$$


$G(t)$

$$
\begin{aligned}
& =\frac{1}{\rho v(t)} \cdot\left[l\left(t, \lambda(t),(\lambda(t))^{\prime}, u(t)+\rho v(t)\right)\right. \\
& \left.-l\left(t, \lambda(t),(\lambda(t))^{\prime}, u(t)\right)\right], \\
& K(T)=\frac{1}{x_{\rho}(T)-x(T)} \\
& \times\left[\Phi\left(x_{\rho}(T),\left(x_{\rho}(T)\right)^{\prime}\right)-\Phi\left(x(T),\left(x_{\rho}\right)^{\prime}(T)\right)\right], \\
& M(T)=\frac{1}{\left(x_{\rho}(T)\right)^{\prime}-(x(T))^{\prime}} \\
& \times\left[\Phi\left(x(T),\left(x_{\rho}(T)\right)^{\prime}\right)-\Phi\left(x(T),(x(T))^{\prime}\right)\right], \\
& A^{l}(t)= \begin{cases}A(t), & x_{\rho}(t)-x(t) \neq 0, \\
0, & \text { otherwise, }\end{cases} \\
& B^{l}(t)= \begin{cases}B(t), & y_{\rho}(t)-y(t) \neq 0, \\
0, & \text { otherwise }\end{cases} \\
& C^{l}(t)= \begin{cases}C(t), & z_{\rho}(t)-z(t) \neq 0, \\
0, & \text { otherwise, }\end{cases} \\
& D^{l}(t)= \begin{cases}D(t), & \left(x_{\rho}(t)\right)^{\prime}-(x(t))^{\prime} \neq 0, \\
0, & \text { otherwise }\end{cases} \\
& E^{l}(t)= \begin{cases}E(t), & \left(y_{\rho}(t)\right)^{\prime}-(y(t))^{\prime} \neq 0, \\
0, & \text { otherwise, }\end{cases} \\
& F^{l}(t)= \begin{cases}F(t), & \left(z_{\rho}(t)\right)^{\prime}-(z(t))^{\prime} \neq 0, \\
0, & \text { otherwise, }\end{cases} \\
& G^{l}(t)= \begin{cases}G(t), & \rho v(t) \neq 0, \\
0, & \text { otherwise, }\end{cases} \\
& \bar{K}^{l}(T)= \begin{cases}K(T), & x_{\rho}(T)-x(T) \neq 0, \\
0, & \text { otherwise, }\end{cases} \\
& \bar{M}^{l}(T)= \begin{cases}M(T), & \left(x_{\rho}(T)\right)^{\prime}-(x(T))^{\prime} \neq 0, \\
0, & \text { otherwise. }\end{cases}
\end{aligned}
$$

From (H5.1) and the fact that $(\widehat{x}(\cdot), \widehat{y}(\cdot), \widehat{z}(\cdot))$ converges to 0 in $M_{\mathbb{F}}^{2}(0, T)$ as $\rho$ tends to 0 , we know that

$$
\begin{aligned}
& \lim _{\rho \rightarrow 0}\left(A^{l}(t)-l_{x}(\theta(t))\right)=0, \\
& \lim _{\rho \rightarrow 0}\left(B^{l}(t)-l_{y}(\theta(t))\right)=0, \\
& \lim _{\rho \rightarrow 0}\left(C^{l}(t)-l_{z}(\theta(t))\right)=0,
\end{aligned}
$$

$$
\begin{aligned}
& \lim _{\rho \rightarrow 0}\left(D^{l}(t)-l_{\tilde{x}}(\theta(t))\right)=0, \\
& \lim _{\rho \rightarrow 0}\left(E^{l}(t)-l_{\tilde{y}}(\theta(t))\right)=0, \\
& \lim _{\rho \rightarrow 0}\left(F^{l}(t)-l_{\tilde{z}}(\theta(t))\right)=0, \\
& \lim _{\rho \rightarrow 0}\left(G^{l}(t)-l_{v}(\theta(t))\right)=0, \\
& 0=\lim _{\rho \rightarrow 0}\left\{\overline { l } \left(t, \Delta x(t), \Delta y(t), \Delta z(t),(\Delta x(t))^{\prime},\right.\right.
\end{aligned}
$$$$
\left.(\Delta y(t))^{\prime},(\Delta z(t))^{\prime}, v(t)\right)
$$$$
-l_{x}(\theta(t)) \Delta x(t)-l_{y}(\theta(t)) \Delta y(t)
$$$$
-l_{z}(\theta(t)) \Delta z(t)-l_{\tilde{x}}(\theta(t))(\Delta x(t))^{\prime}
$$$$
-l_{\tilde{y}}(\theta(t))(\Delta y(t))^{\prime}
$$$$
\left.-l_{\widetilde{z}}(\theta(t))(\Delta z(t))^{\prime}-l_{v}(\theta(t)) v(t)\right\},
$$

where $\theta(t)=\left(t, x(t), y(t), z(t), \quad(x(t))^{\prime}, \quad(y(t))^{\prime}, \quad(z(t))^{\prime}\right.$, $u(t)) . \Delta y(T)$ has similar results.

As we know, (57) has a unique solution $\left(x^{1}(\cdot)\right.$, $\left.y^{1}(\cdot), z^{1}(\cdot)\right)$. Therefore, the solution $(\Delta x(\cdot), \Delta y(\cdot), \Delta z(\cdot))$ converges to $\left(x^{1}(\cdot), y^{1}(\cdot), z^{1}(\cdot)\right)$ in $M_{\mathbb{F}}^{2}\left(0, T ; \mathbb{R}^{n+m+m \times d}\right)$ as $\rho$ tends to 0 .

Because $u(\cdot)$ is an optimal control, then

$$
\rho^{-1}[J(u(\cdot)+\rho v(\cdot))-J(u(\cdot))] \geq 0 .
$$

From (65) and Lemma 15, we have the following.

Lemma 16. One supposes that (H5.1) holds. Then, the following variational inequality holds:

$$
\begin{gathered}
0 \leq E \int_{0}^{T} E^{\prime}\left[L_{x}(\theta(t)) x^{1}(t)+L_{y}(\theta(t)) y^{1}(t)\right. \\
+L_{z}(\theta(t)) z^{1}(t)+L_{\tilde{x}}(\theta(t))\left(x^{1}(t)\right)^{\prime} \\
+L_{\tilde{y}}(\theta(t))\left(y^{1}(t)\right)^{\prime} \\
\left.+L_{\widetilde{z}}(\theta(t))\left(z^{1}(t)\right)^{\prime}+L_{v}(\theta(t)) v(t)\right] d t \quad(66) \\
+E\left[E ^ { \prime } \left[\varphi_{x}\left(x(T),(x(T))^{\prime}\right) x^{1}(T)\right.\right. \\
\left.+\varphi_{\tilde{x}}\left(x(T),(x(T))^{\prime}\right)\left(x^{1}(T)\right)^{\prime}\right] \\
\left.+h_{y}(y(0)) y^{1}(0)\right]
\end{gathered}
$$

where $\theta(t)=\left(t, x(t), y(t), z(t),(x(t))^{\prime},(y(t))^{\prime},(z(t))^{\prime}, u(t)\right)$. 
Proof. Let $\rho \rightarrow 0$ in (65); from Lemma 15 and (H5.1), it is obvious that

$$
\begin{aligned}
& \rho^{-1} E\left(E ^ { \prime } \left[\varphi\left(x_{\rho}(T)\right),\left(x_{\rho}(T)^{\prime}\right)\right.\right. \\
& \left.\left.-\varphi\left(x(T),(x(T))^{\prime}\right)\right]\right) \\
& \longrightarrow E E^{\prime}\left[\varphi_{x}\left(x(T),(x(T))^{\prime}\right) x^{1}(T)\right. \\
& \left.+\varphi_{\tilde{x}}\left(x(T),(x(T))^{\prime}\right)\left(x^{1}(T)\right)^{\prime}\right] ; \\
& \rho^{-1} E\left[h\left(y_{\rho}(0)\right)-h(y(0))\right] \longrightarrow E\left[h_{y}(y(0)) y^{1}(0)\right] ; \\
& \rho^{-1} E \int_{0}^{T} E^{\prime}\left[L\left(\chi_{\rho}(t), u(t)+\rho v(t)\right)-L(\theta(t))\right] d t \\
& \longrightarrow E \int_{0}^{T} E^{\prime}\left[L_{x}(\theta(t)) x^{1}(t)\right. \\
& +L_{y}(\theta(t)) y^{1}(t)+L_{z}(\theta(t)) z^{1}(t) \\
& +L_{\tilde{x}}(\theta(t))\left(x^{1}(t)\right)^{\prime} \\
& +L_{\tilde{y}}(\theta(t))\left(y^{1}(t)\right)^{\prime}+L_{\widetilde{z}}(\theta(t))\left(z^{1}(t)\right)^{\prime} \\
& \left.+L_{v}(\theta(t)) v(t)\right] d t .
\end{aligned}
$$

The proof is complete.

Now we introduce the following adjoint mean-field FBSDE to (57):

$$
\begin{aligned}
& d p(t)=E^{\prime}\left[g_{y}^{T}(\theta(t)) p(t)-f_{y}^{T}(\theta(t)) q(t)\right. \\
& -\sigma_{y}^{T}(\theta(t)) k(t)-L_{y}(\theta(t)) \\
& +g_{\tilde{y}}^{T}(\varrho(t))(p(t))^{\prime} \\
& -f_{\tilde{y}}^{T}(\varrho(t))(q(t))^{\prime}-\sigma_{\tilde{y}}^{T}(\varrho(t))(k(t))^{\prime} \\
& \left.-L_{\tilde{y}}(\varrho(t))\right] d t \\
& +E^{\prime}\left[g_{z}^{T}(\theta(t)) p(t)-f_{z}^{T}(\theta(t)) q(t)\right. \\
& -\sigma_{z}^{T}(\theta(t)) k(t)-L_{z}(\theta(t)) \\
& +g_{\tilde{z}}^{T}(\varrho(t))(p(t))^{\prime} \\
& -f_{\tilde{z}}^{T}(\varrho(t))(q(t))^{\prime}-\sigma_{\widetilde{z}}^{T}(\varrho(t))(k(t))^{\prime} \\
& \left.-L_{\widetilde{z}}(\varrho(t))\right] d B_{t} \\
& -d q(t)=E^{\prime}\left[-g_{x}^{T}(\theta(t)) p(t)+f_{x}^{T}(\theta(t)) q(t)\right. \\
& +\sigma_{x}^{T}(\theta(t)) k(t)
\end{aligned}
$$

$$
\begin{aligned}
& +L_{x}(\theta(t))-g_{\tilde{x}}^{T}(\varrho(t))(p(t))^{\prime} \\
& +f_{\tilde{x}}^{T}(\varrho(t))(q(t))^{\prime} \\
& +\sigma_{\tilde{x}}^{T}(\varrho(t))(k(t))^{\prime} \\
& \left.+L_{\tilde{x}}(\varrho(t))\right] d t-k(t) d B_{t}
\end{aligned}
$$$$
p(0)=-h_{y}(y(0)) \text {, }
$$$$
q(T)=E^{\prime}\left[\varphi_{x}\left(x(T),(x(T))^{\prime}\right)\right.
$$$$
+\varphi_{\tilde{x}}\left((x(T))^{\prime}, x(T)\right)
$$$$
-\Phi_{x}\left(x(T), x^{\prime}(T)\right) p(T)
$$$$
\left.-\Phi_{\widetilde{x}}\left(x^{\prime}(T), x(T)\right)(p(T))^{\prime}\right],
$$

where $\theta(t)=\left(t, x(t), y(t), z(t),(x(t))^{\prime},(y(t))^{\prime},(z(t))^{\prime}\right.$, $u(t))$ and $\varrho(t)=\left(t,(x(t))^{\prime},(y(t))^{\prime},(z(t))^{\prime}, x(t), y(t), z(t)\right.$, $\left.(u(t))^{\prime}\right)$. From Theorem 6 , we know that there exists a unique triple $(p(\cdot), q(\cdot), k(\cdot))$ satisfying $(68)$.

We define the Hamiltonian function $H$ as follows:

$$
\begin{aligned}
& H(t, x, y, z, \tilde{x}, \tilde{y}, \tilde{z}, v, p, q, k) \\
&=\langle p,-g(t, x, y, z, \tilde{x}, \tilde{y}, \tilde{z}, v)\rangle \\
&+\langle q, f(t, x, y, z, \tilde{x}, \tilde{y}, \tilde{z}, v)\rangle \\
&+\langle k, \sigma(t, x, y, z, \tilde{x}, \tilde{y}, \tilde{z}, v)\rangle \\
&+L(t, x, y, z, \tilde{x}, \tilde{y}, \tilde{z}, v) .
\end{aligned}
$$

Then we have the following maximum principle.

Theorem 17. Let $u(\cdot)$ be an optimal control and let $(x(\cdot)$, $y(\cdot), z(\cdot))$ be the corresponding trajectory. Then, one has

$$
\begin{aligned}
& E^{\prime}\left\langle H_{v}(t, \chi(t), u(t), p(t), q(t), k(t)), v-u(t)\right\rangle \\
& \quad \geq 0, \quad \forall v \in U, d t d P \text {-a.e., }
\end{aligned}
$$

where $\chi(t)=\left(x(t), y(t), z(t),(x(t))^{\prime},(y(t))^{\prime},(z(t))^{\prime}\right),(p, q, k)$ is the solution of the adjoint (68).

Proof. Applying Itô's formula to $\left\langle x^{1}(t), q(t)\right\rangle+\left\langle y^{1}(t), p(t)\right\rangle$, from (57) and (68) and (H3.1), (H3.2), (H3.3), and (H5.1), with the help of (66) and (69), for $v(\cdot)$ such that $u(\cdot)+v(\cdot) \epsilon$ $\mathcal{U}_{\text {ad }}$, we get

$$
E \int_{0}^{T} E^{\prime}\left\langle H_{v}(t, \chi(t), u(t), p(t), q(t), k(t)), v\right\rangle d t \geq 0 .
$$

Therefore, we have

$$
\begin{aligned}
& E^{\prime}\left\langle H_{v}(t, \chi(t), u(t), p(t), q(t), k(t)), v-u(t)\right\rangle \\
& \quad \geq 0, \quad \forall v \in U \text {, a.s. a.e. }
\end{aligned}
$$




\section{Application to the Mean-Field LQ Problems}

In this section, we consider a linear-quadratic control problem as an example. For simplicity, we only consider onedimensional case; that is, $m=n=d=1$. The state equation can be written as follows:

$$
\begin{gathered}
d x(t)=E^{\prime}\left[a_{1}(x(t))^{\prime}+a_{2} x(t)\right. \\
\left.-a_{3}\left(a_{3} y(t)+a_{4} z(t)\right)+\alpha v(t)\right] d t \\
+E^{\prime}\left[a_{5}(x(t))^{\prime}+a_{6} x(t)\right. \\
\left.-a_{4}\left(a_{3} y(t)+a_{4} z(t)\right)+\beta v(t)\right] d B_{t}, \\
-d y(t)=E^{\prime}\left[a_{1}(y(t))^{\prime}+a_{2} y(t)+a_{5}(z(t))^{\prime}\right. \\
\left.\quad+a_{6} z(t)+a_{7} x(t)+\gamma v(t)\right] d t-z(t) d B_{t}, \\
x(0)=a, \quad y(T)=\eta,
\end{gathered}
$$

where the constants $a_{i}, i=1, \ldots, 7, \alpha, \beta, \gamma$ are positive and $v \in \mathcal{U}$. The cost functional is

$$
\begin{aligned}
& J(v(\cdot)) \\
& =\frac{1}{2} E E^{\prime}\left[\int_{0}^{T}\left[M(t) y^{2}(t)+R(t) z^{2}(t)+S(t) u^{2}(t)\right] d t\right. \\
& \left.+Q y^{2}(0)\right]
\end{aligned}
$$

where $M(t), R(t), Q$ are bounded and nonnegative and $S(t)$ is bounded and positive. Then, the adjoint equation is the following mean-field FBSDE:

$$
\begin{gathered}
d p(t)=E^{\prime}\left[a_{2} p(t)+a_{3}^{2} q(t)+a_{3} a_{4} k(t)\right. \\
\left.-M(t) y(t)+a_{1}(p(t))^{\prime}\right] d t \\
+E^{\prime}\left[a_{6} p(t)+a_{3} a_{4} q(t)\right. \\
\left.+a_{4}^{2} k(t)-R(t) z+a_{5}(p(t))^{\prime}\right] d B_{t}, \\
-d q(t)=E^{\prime}\left[-a_{7} p(t)+a_{2} q(t)+a_{6} k(t)\right. \\
\left.+a_{1} q^{\prime}(t)+a_{5}(k(t))^{\prime}\right] d t \\
-k(t) d B_{t}, \\
p(0)=-Q y(0), \quad q(T)=0 .
\end{gathered}
$$

Let

$$
\begin{aligned}
& H(t, x, y, z, \tilde{x}, \tilde{y}, \tilde{z}, v, p, q, k) \\
&=-p\left(a_{1} \tilde{y}+a_{2} y+a_{5} \tilde{z}+a_{6} z+a_{7} x+\gamma v\right) \\
&+q\left(a_{1} \tilde{x}+a_{2} x-a_{3}\left(a_{3} y+a_{4} z\right)+\alpha v\right) \\
&+k\left(a_{5} \tilde{x}+a_{6} x-a_{4}\left(a_{3} y+a_{4} z\right)+\beta v\right) \\
&+\frac{1}{2}\left(M(t) y^{2}(t)+R(t) z^{2}(t)+S(t) u^{2}(t)\right) .
\end{aligned}
$$

Then, from the stochastic maximum principle (Theorem 17), we have

$$
-p(t) \gamma+q(t) \alpha+k(t) \beta+S(t) u^{*}(t)=0 ;
$$

that is,

$$
u^{*}(t)=-S^{-1}(t)(-p(t) \gamma+q(t) \alpha+k(t) \beta) .
$$

However, the maximum principle gives only the necessary condition for optimal control.

Now we prove that $u^{*}$ is the optimal control. For all $v \in \mathcal{U}$, let $\left(x^{v}(\cdot), y^{v}(\cdot), z^{v}(\cdot)\right)$ be the corresponding trajectory. Then

$$
\begin{gathered}
J(v(\cdot))-J\left(u^{*}(\cdot)\right) \\
=\frac{1}{2} E E^{\prime}\left[\int _ { 0 } ^ { T } \left[M(t)\left(\left(y^{v}(t)\right)^{2}-\left(y^{*}(t)\right)^{2}\right)\right.\right. \\
+R(t)\left(\left(z^{v}(t)\right)^{2}-\left(z^{*}(t)\right)^{2}\right) \\
\left.+S(t)\left((v(t))^{2}-\left(u^{*}(t)\right)^{2}\right)\right] d t \\
\left.+Q\left(\left(y^{v}(0)\right)^{2}-\left(y^{*}(0)\right)^{2}\right)\right] \\
\geq E E^{\prime} \int_{0}^{T}\left[M(t) y^{*}(t)\left(y^{v}(t)-y^{*}(t)\right)\right. \\
+R(t) z^{*}(t)\left(z^{v}(t)-z^{*}(t)\right) \\
\left.+S(t) u^{*}(t)\left(v(t)-u^{*}(t)\right)\right] d t \\
+Q y^{*}(0)\left(y^{v}(0)-y^{*}(0)\right) .
\end{gathered}
$$

We apply Itô's formula to $p(t)\left(y^{v}(t)-y^{*}(t)\right)+q(t)\left(x^{v}(t)-\right.$ $\left.x^{*}(t)\right)$, where $(p(t), q(t), k(t))$ is the solution of adjoint equation with the state process $\left(x^{*}(t), y^{*}(t), z^{*}(t)\right)$; notice that when $c$ is a constant, then $E^{\prime}\left[c X(t)(X(t))^{\prime}\right]=$ $E E^{\prime}\left[c(X(t))^{\prime} X(t)\right]$ and we get

$$
\begin{gathered}
E E^{\prime}\left[Q y^{*}(0)\left(y^{v}(0)-y^{*}(0)\right)\right] \\
=E E^{\prime} \int_{0}^{T}\left[(-p(t) \gamma+q(t) \alpha+k(t) \beta)\left(v(t)-u^{*}(t)\right)\right. \\
-M(t) y^{*}(t)\left(y^{v}(t)-y^{*}(t)\right) \\
\left.-R(t) z^{*}(t)\left(z^{v}(t)-z^{*}(t)\right)\right] d t .
\end{gathered}
$$


Therefore, from the definition of $u^{*}(t)$,

$$
\begin{aligned}
& J(v(\cdot))-J\left(u^{*}(\cdot)\right) \\
& \geq E E^{\prime}\left[\int_{0}^{T}\left(-p(t) \gamma+q(t) \alpha+k(t) \beta+S(t) u^{*}(t)\right)\right. \\
& \left.\quad \times\left(v(t)-u^{*}(t)\right)\right] d t=0,
\end{aligned}
$$

for any $v \in \mathcal{U}$. It means that $u^{*}(t)$ is an optimal control.

Remark 18. Under our assumption, the existence and the uniqueness of the solution of (73) and (75) can be obtained by combining the method of Theorem 3.1 and Theorem 2.1 in [6]. We omit the proof.

\section{Conflict of Interests}

The authors declare that there is no conflict of interests regarding the publication of this paper.

\section{Acknowledgments}

The work has been supported by the NSF of China (nos. 11071144, 11171187, and 11222110), Shandong Province (nos. BS2011SF010 and JQ201202), Program for New Century Excellent Talents in University (no. NCET-12-0331), and the 111 Project (no. B12023).

\section{References}

[1] E. Pardoux and S. Peng, "Adapted solution of a backward stochastic differential equation," Systems \& Control Letters, vol. 14, no. 1, pp. 55-61, 1990.

[2] F. Antonelli, "Backward-forward stochastic differential equations," The Annals of Applied Probability, vol. 3, no. 3, pp. 777793, 1993.

[3] J. Ma, P. Protter, and J. Yong, "Solving forward-backward stochastic differential equations explicitly-a four step scheme," Probability Theory and Related Fields, vol. 98, no. 3, pp. 339-359, 1994.

[4] Y. Hu and S. Peng, "Solution of forward-backward stochastic differential equations," Probability Theory and Related Fields, vol. 103, no. 2, pp. 273-283, 1995.

[5] E. Pardoux and S. Tang, "Forward-backward stochastic differential equations and quasilinear parabolic PDEs," Probability Theory and Related Fields, vol. 114, no. 2, pp. 123-150, 1999.

[6] S. Peng and Z. Wu, "Fully coupled forward-backward stochastic differential equations and applications to optimal control," SIAM Journal on Control and Optimization, vol. 37, no. 3, pp. 825-843, 1999.

[7] J. M. Yong, "Forward-backward stochastic differential equations with mixed initial-terminal conditions," Transactions of the American Mathematical Society, vol. 362, no. 2, pp. 1047-1096, 2010.

[8] F. Delarue and S. Menozzi, "A forward-backward stochastic algorithm for quasi-linear PDEs," The Annals of Applied Probability, vol. 16, no. 1, pp. 140-184, 2006.
[9] D. Zhang, "Forward-backward stochastic differential equations and backward linear quadratic stochastic optimal control problem," Communications in Mathematical Research, vol. 25, no. 5, pp. 402-410, 2009.

[10] J. Ma, Z. Wu, D. Z. Zhang, and J.-F. Zhang, "On wellposedness of forward-backward SDEs-a unified approach," http:// arxiv.org/abs/1110.4658.

[11] J. Ma and J. M. Yong, Forward-Backward Stochastic Differential Equations and Their Applications, Springer, Berlin, Germany, 1999.

[12] L. S. Pontryagin, V. G. Boltanskii, and R. V. Gamkrelidze, The Mathematical Theory of Optimal Processes, Interscience, New York, NY, USA, 1962.

[13] R. Bellman, Dynamic Programming, Princeton University Press, Princeton, NJ, USA, 1957.

[14] H. J. Kushner, "On the stochastic maximum principle: fixed time of control," Journal of Mathematical Analysis and Applications, vol. 11, pp. 78-92, 1965.

[15] H. J. Kushner, "Necessary conditions for continuous parameter stochastic optimization problems," SIAM Journal on Control and Optimization, vol. 10, no. 3, pp. 550-565, 1972.

[16] A. Bensoussan, "Lectures on stochastic control," in Nonlinear Filtering and Stochastic Control, vol. 972 of Lecture Notes in Mathematics, pp. 1-62, Springer, Berlin, Germany, 1982.

[17] U. G. Haussmann, A Stochastic Maximum Principle for Optimal Control of Diffusions, Longman Scientific \& Technical, Essex, UK, 1986.

[18] S. G. Peng, "A general stochastic maximum principle for optimal control problems," SIAM Journal on Control and Optimization, vol. 28 , no. 4, pp. 966-979, 1990.

[19] Z. Wu, "Maximum principle for optimal control problem of fully coupled forward-backward stochastic systems," Systems Science and Mathematical Sciences, vol. 11, no. 3, pp. 249-259, 1998.

[20] R. Buckdahn, J. Li, and S. Peng, "Mean-field backward stochastic differential equations and related partial differential equations," Stochastic Processes and their Applications, vol. 119, no. 10, pp. 3133-3154, 2009.

[21] R. Buckdahn, B. Djehiche, J. Li, and S. Peng, "Mean-field backward stochastic differential equations: a limit approach," The Annals of Probability, vol. 37, no. 4, pp. 1524-1565, 2009.

[22] D. Andersson and B. Djehiche, "A maximum principle for SDEs of mean-field type," Applied Mathematics \& Optimization, vol. 63, no. 3, pp. 341-356, 2011.

[23] R. Buckdahn, B. Djehiche, and J. Li, "A general stochastic maximum principle for SDEs of mean-field type," Applied Mathematics \& Optimization, vol. 64, no. 2, pp. 197-216, 2011.

[24] J. Li, "Stochastic maximum principle in the mean-field controls," Automatica, vol. 48, no. 2, pp. 366-373, 2012.

[25] A. Bensoussan, K. C. J. Sung, S. C. P. Yam, and S. P. Yung, "Linear-quadratic mean field games".

[26] Y. L. Qin, Mean-field forward-backward stochastic differential equations and related questions [M.S. thesis], Shandong University, Weihai, China, 2012. 


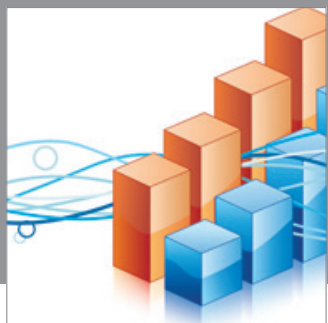

Advances in

Operations Research

mansans

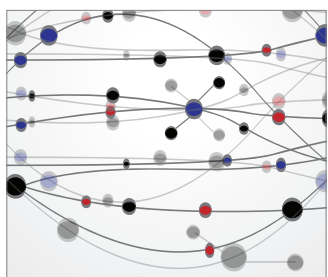

The Scientific World Journal
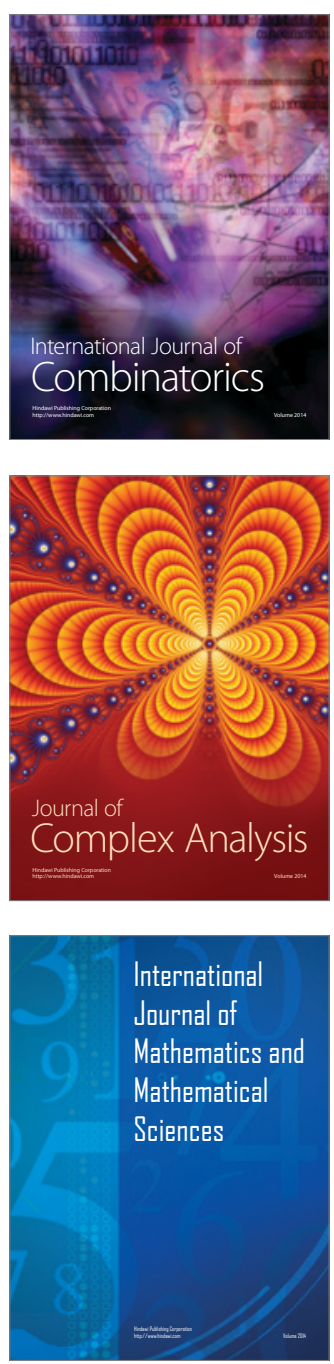
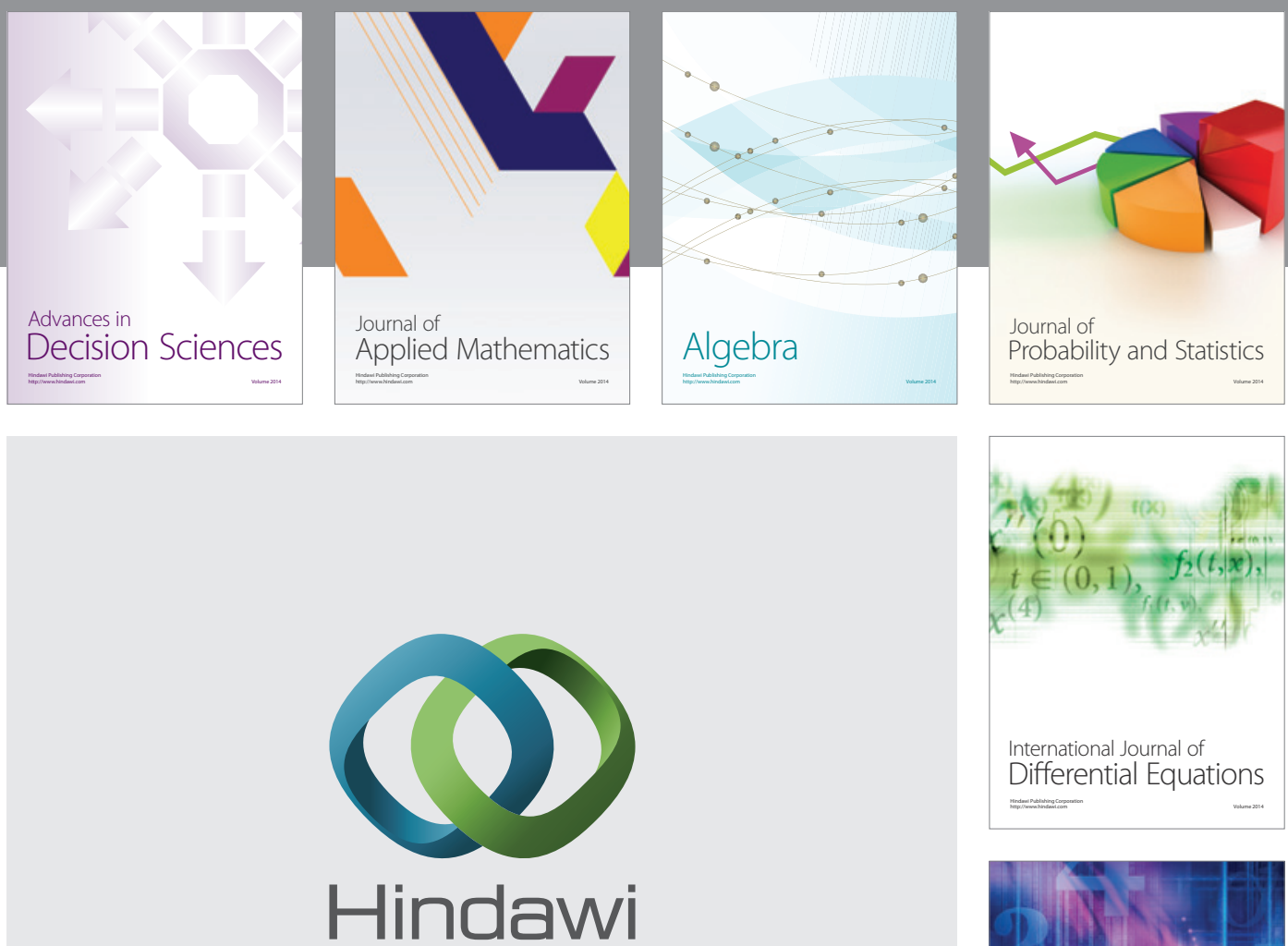

Submit your manuscripts at http://www.hindawi.com
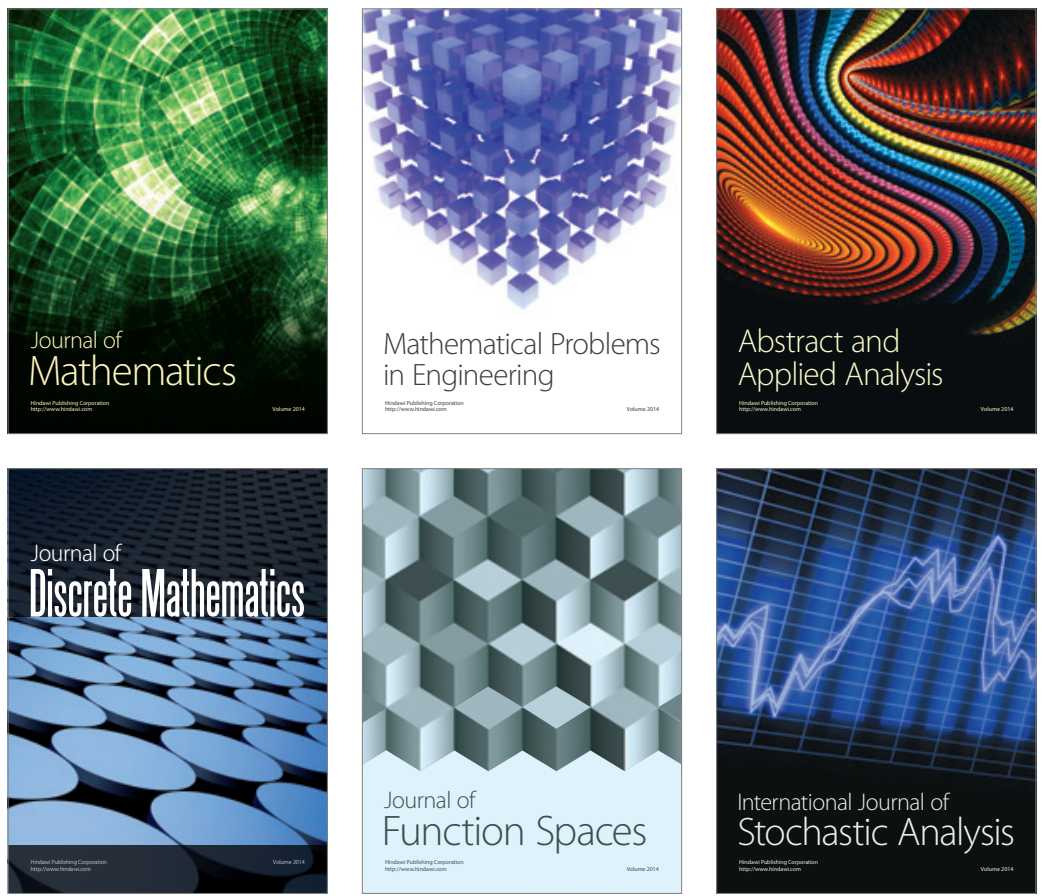

Journal of

Function Spaces

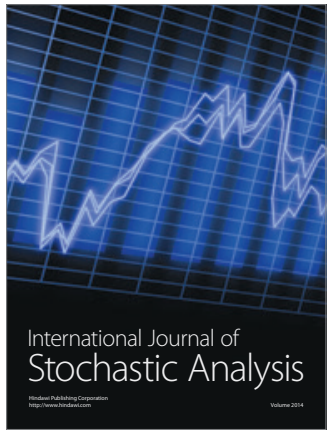

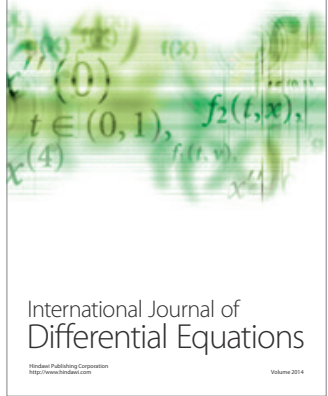
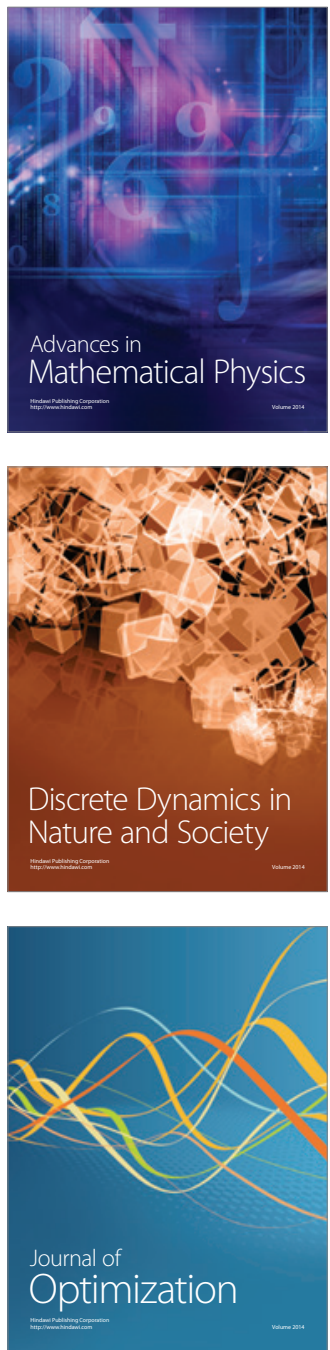